\title{
Modelling Unsaturated Flow in Porous Media Using an Improved Picard Iteration Scheme
}

\section{S.R. Zhu}

Chengdu University of Technology

\section{L.Z. Wu ( $13880936151 @ 163 . c o m$ )}

Chengdu University of Technology

\section{T. Ma}

Chengdu University of Technology

S.H. Li

Chengdu University of Technology

\section{Research Article}

Keywords: Unsaturated flow, Picard iteration, Convergence rate, Adaptive relaxation, Multistep preconditioner

Posted Date: May 19th, 2021

DOI: https://doi.org/10.21203/rs.3.rs-483811/v1

License: (c) (1) This work is licensed under a Creative Commons Attribution 4.0 International License. Read Full License 
Modelling unsaturated flow in porous media using an improved

\title{
Picard iteration scheme
}

\author{
S. R. Zhu ${ }^{\text {a }}$ L. Z. Wu ${ }^{\text {a* }}$, T. Ma ${ }^{\text {a }}$, S. H. Li ${ }^{\text {a }}$
}

a) State Key Laboratory of Geohazard Prevention and Geoenvironment Protection, Chengdu University of Technology, 610059, Chengdu, Sichuan, P. R. China

*corresponding author: Email: wulizhou07@.cdut.edu.cn

Submitted on April 30, 2021 
Abstract: The numerical solution of various systems of linear equations describing fluid infiltration uses the Picard iteration (PI). However, because many such systems are ill-conditioned, the solution process often has a poor convergence rate, making it very time-consuming. In this study, a control volume method based on non-uniform nodes is used to discretize the Richards equation, and adaptive relaxation is combined with a multistep preconditioner to improve the convergence rate of PI. The resulting adaptive relaxed PI with multistep preconditioner (MP $(m)$-ARPI) is used to simulate unsaturated flow in porous media. Three examples are used to verify the proposed schemes. The results show that $\operatorname{MP}(m)$-ARPI can effectively reduce the condition number of the coefficient matrix for the system of linear equations. Compared with conventional PI, MP(m)-ARPI achieves faster convergence, higher computational efficiency, and enhanced robustness. These results demonstrate that improved scheme is an excellent prospect for simulating unsaturated flow in porous media.

Keywords: Unsaturated flow; Picard iteration; Convergence rate; Adaptive relaxation; Multistep preconditioner. 


\section{Introduction}

Many geotechnical engineering problems involve unsaturated seepage, such as the soil slope stability and dam infiltration involved in rainfall or groundwater changes, and the transportation of pollutants in landfill (Morway et al., 2013; Ku et al., 2017; Li et al., 2020). Therefore, effectively simulating and analyzing unsaturated seepage is of great practical significance (Wang et al., 2011; Zang et al., 2016; Wu et al., 2016; 2020a). The Richards equation (RE) provides a basic description of unsaturated infiltration (Richards, 1931), although analytical solutions to the RE are difficult to derive. Srivastava and Yeh (1991) used a Laplace transformation technique to obtain analytical solutions of the transient flows in homogeneous and two-layer soils, while Tracy (2006) proposed two- and three-dimensional analytical solutions of the RE using simple exponential models. Although their actual applicability is limited, these are good references for theoretical analysis (Zambra et al., 2012; Liu et al., 2015; Baron et al., 2017).

Generally, the RE must be solved numerically by a finite difference scheme (Wang and Anderson, 1982), finite element technique (Šimůnek et al., 2009), or finite volume method (Patankar, 1980) combined with an effective iterative scheme (Solin and Kuraz, 2011; Zha et al., 2013; Brenner and Cancès, 2017). Recently, Chávez-Negrete et al. (2018) proposed a generalized finite difference method and an adaptive step-size Crank-Nicolson method to obtain more accurate numerical solutions to the RE. Dolejší et al. (2019) proposed an adaptive higher-order space-time discontinuous-Galerkin ( $h p$ STDG) method to obtain highly efficient and accurate solutions to the RE. Svyatskiy 
and Lipnikov (2017) proposed a second-order-accurate finite volume scheme that solves the RE using high-order upwind algorithms for the relative permeability. Additionally, other advanced numerical methods for solving variable saturated flow problems exhibit high levels of accuracy, computational efficiency, or ease of implementation under certain conditions. These include the two-level adaptive domain decomposition (Kuraz et al., 2015), finite analytic method (Zhang et al., 2016), and Chebyshev's spectral method (Wu et al., 2020b). A system of linear equations derived from the RE need to be solved iteratively, such as by using the Picard iteration (PI) scheme. Because hydraulic conductivity and water content are nonlinear functions of the pressure head, the numerical results to the RE using PI are relatively unreliable. A modified PI could ensure the mass conservation in the temporal discretization (Celia et al., 1990). Recently, Zha et al. (2017) further improved the algorithms of Celia et al. (1990) to overcome the numerical divergence that occurs when simulating infiltration into extremely dry soil. However, these improvements are limited to the overall mass balance of the RE, and do not improve the convergence rate of the solutions.

As PI has poor convergence and is time-consuming, increasing attention has focused on improving its convergence rate (Durbin et al., 2007; Lott et al., 2012; List and Radu, 2016; Illiano et al., 2020). Introducing a relaxation process can improve the convergence rate of PI (Paniconi and Putti, 1994), but determining the optimal relaxation factor is difficult. Durbin et al. (2007) proposed an adaptive under-relaxation of PI to accelerate the convergence rate for nonlinear groundwater flow problems. Later, De Smedt et al. (2010) developed an unstable manifold correction method for the 
adaptive relaxation of PI to solve the velocity field in higher-order ice-flow models. Although the convergence rate of PI can be improved by adaptive relaxation techniques, the numerical results are not very robust. Preprocessing can improve the ill-conditioned nature of the associated systems of linear equations; that is, reducing the condition number of the coefficient matrix results in faster convergence (Benzi, 2002). Recently, Wang and Zhang (2003) developed a class of parallel multistep successive preconditioning strategies that significantly improve the computational efficiency and stability of the solution process for systems of linear equations. Liu et al. (2015) used a newly developed Jacobian iteration method to overcome the non-convergence for solving the infiltration problem in layered soils. Zhu et al. (2020) used an improved Chebyshev semi-iterative method to obtain a faster convergence rate for solving the RE. The preprocessing method can effectively improve the ill-condition of the system of linear equations, thereby improving the convergence rate. Thus, to enhance the computational efficiency of PI, this paper proposes an adaptive relaxed PI scheme with a multistep preconditioner (MP $(m)$-ARPI) to simulate unsaturated flow. The purpose of this paper is to develop a new iteration method for the efficient solution of one- and two-dimensional infiltration equations. $\mathrm{MP}(m)$-ARPI is applied to investigate twodimensional (2D) transient flows in homogeneous porous media. Three examples of unsaturated flow are employed to examine the proposed schemes. The convergence rate, calculation efficiency, calculation accuracy, and robustness of the proposed schemes are compared and discussed against the numerical results of conventional iteration methods. 


\section{Numerical Procedures}

The RE describes multidimensional unsaturated flow (Wu et al., 2013; Duc et al., 2020). The 2D RE is written as:

$$
\frac{\partial}{\partial x}\left[K_{x}(h) \frac{\partial h}{\partial x}\right]+\frac{\partial}{\partial z}\left[K_{z}(h)\left(\frac{\partial h}{\partial z}+1\right)\right]=\frac{\partial \theta}{\partial t},
$$

where $z$ and $x$ are the vertical and horizontal coordinates, respectively; $h$ represents the pressure head; $\theta$ denotes the moisture content; $K_{x}(h)$ and $K_{z}(h)$ represent hydraulic conductivity along the $x$-axis and the $z$-axis, respectively, and are both functions of the pressure head $(h)$. For one-dimensional (1D) problems, Eq. (1) can be simplified as:

$$
\frac{\partial}{\partial z}\left[K_{z}(h)\left(\frac{\partial h}{\partial z}+1\right)\right]=\frac{\partial \theta}{\partial t},
$$

where $\theta$ and $K(h)$ can be described using exponential models (Gardner, 1958), in which

$$
\begin{gathered}
\theta(h)=\theta_{r}+\left(\theta_{s}-\theta_{r}\right) e^{\alpha h}, \\
K(h)=K_{s} e^{\alpha h},
\end{gathered}
$$

where $K_{s}$ is the saturated hydraulic conductivity; $\theta_{s}$ and $\theta_{r}$ represent the saturated and residual moisture content, respectively; and $\alpha$ is the fitting parameter. Furthermore, the control volume method (Patankar, 1980) can be used to discretize Eqs. (1) and (2). A 1D infiltration model is illustrated in Fig. 1. Firstly, the nonuniform grid coordinates can be expressed as:

$$
z_{i}=\cos (i \pi / N) \times \frac{L}{2}+\frac{L}{2}, \quad i=N, N-1, \mathrm{~K} 0,
$$

where $i$ denotes the nonuniform grid nodes; $L$ is the height of the soil layer; and $N$ 
represents the number of nodes. To ensure the mass balance in the temporal discretization, the following modified iteration format for $\partial \theta / \partial t$ is proposed (Celia et al., 1990):

$$
\frac{\partial \theta}{\partial t}=C^{j+1, k} \frac{h^{j+1, k+1}-h^{j+1, k}}{\Delta t}+\frac{\theta^{j+1, k}-\theta^{j}}{\Delta t}
$$

where $C$ is the specific moisture capacity, defined as $C(h)=\partial \theta / \partial h ; \Delta t$ denotes the time step; and the superscripts $j$ and $k$ represent the time step and number of iterations, respectively. Equation (6) has been widely used in commercial software such as HYDRUS (Šimůnek et al., 2009; Zha et al., 2017).

The discretized equation is now derived by integrating Eq. (2) over the control volume in Fig. 1 and over the time interval from $t$ to $t+\Delta t$ (Patankar, 1980). Thus:

$$
\int_{i_{W}}^{i_{E}} \int_{t}^{t+\Delta t} \frac{\partial \theta}{\partial t} d t d z=\int_{t}^{t+\Delta t} \int_{i_{W}}^{i_{E}} \frac{\partial}{\partial z}\left[K_{z}(h)\left(\frac{\partial h}{\partial z}+1\right)\right] d z d t
$$

Substituting Eq. (6) into Eq. (7), we obtain:

$$
\begin{aligned}
& C_{i}^{j+1, k} \frac{h_{i}^{j+1, k+1}-h_{i}^{j+1, k}}{\Delta t}(\Delta z)_{i}+\frac{\theta_{i}^{j+1, k}-\theta_{i}^{j}}{\Delta t}(\Delta z)_{i}=\frac{K_{i_{E}}^{j+1, k}\left(h_{i+1}^{j+1, k+1}-h_{i}^{j+1, k+1}\right)}{(\delta z)_{E}} \\
& -\frac{K_{i_{W}}^{j+1, k}\left(h_{i}^{j+1, k+1}-h_{i-1}^{j+1, k+1}\right)}{(\delta z)_{W}}+K_{i_{E}}^{j+1, k}-K_{i_{W}}^{j+1, k}
\end{aligned},
$$

where:

$$
\begin{gathered}
K_{i_{E}}=\frac{2 K_{i} K_{i+1}}{K_{i}+K_{i+1}} \\
K_{i_{W}}=\frac{2 K_{i} K_{i-1}}{K_{i}+K_{i-1}}
\end{gathered}
$$

For steady-state seepage, Eq. (8) can be simplified as: 


$$
\frac{K_{i_{E}}^{k}\left(h_{i+1}^{k+1}-h_{i}^{k+1}\right)}{(\delta z)_{E}}-\frac{K_{i_{W}}^{k}\left(h_{i}^{k+1}-h_{i-1}^{k+1}\right)}{(\delta z)_{W}}+K_{i_{E}}^{k}-K_{i_{W}}^{k}=0 .
$$

According to Eqs. (8) and (10), the matrix format of PI based on a nonuniform grid can be written as:

$$
\begin{gathered}
\mathbf{A}\left(\mathbf{h}^{j+1, k}\right) \mathbf{h}^{j+1, k+1}=\mathbf{f}\left(\mathbf{h}^{j+1, k}, \mathbf{h}^{j}\right) . \\
\mathbf{A}\left(\mathbf{h}^{k}\right) \mathbf{h}^{k+1}=\mathbf{f}\left(\mathbf{h}^{k}\right) .
\end{gathered}
$$

where $\mathbf{A}$ is a symmetrical tridiagonal coefficient matrix; $\mathbf{h}$ and $\mathbf{f}$ are vectors. A system of linear algebraic equations is first derived at each iteration, and then a basic linear iterative method (such as Gaussian elimination, conjugate gradient method, or the Gauss-Seidel method) is used to solve the linear equations (Šimůnek et al., 2009). As PI determines the computational efficiency, we use the Gauss-Seidel method to solve the system of linear equations at each iteration.

After solving Eqs. (11) and (12) for the first time, the coefficients in these equations are re-evaluated using the first solution, and the new system of linear equations is again solved. The iteration procedure terminates when the iteration error in terms of the $l_{\infty}$ norm satisfies the following:

$$
\frac{\left\|\mathbf{h}^{j, k+1}-\mathbf{h}^{j, k}\right\|_{\infty}}{\left\|\mathbf{h}^{j, k}\right\|_{\infty}}<\varepsilon
$$

where $\varepsilon$ is the tolerance (set to $10^{-8}$ in this paper).

\section{Improved PI Scheme}

PI has a first-order convergence rate with poor calculation efficiency. To improve the convergence rate of $\mathrm{PI}$, adaptive relaxation and multistep preconditioner strategies are 
introduced. First, when $k>1$ in Eqs. (11) and (12), the following adaptive relaxation method is adopted:

$$
\mathbf{h}^{k+1} \leftarrow \mathbf{h}^{k}+\lambda_{k}\left(\mathbf{h}^{k+1}-\mathbf{h}^{k}\right) ; \quad \lambda_{k} \in(0,2)
$$

where $\lambda_{k}$ is the $k$ th relaxation factor, which is adjusted according to the generalized angle $\varphi$ between the current iteration increment $\Delta \mathbf{h}$ and the previous iteration increment $\Delta \overline{\mathbf{h}}$ as follows:

$$
\varphi=\arccos \left(\frac{\Delta \mathbf{h} g \Delta \overline{\mathbf{h}}}{\|\Delta \mathbf{h}\|\|\Delta \overline{\mathbf{h}}\|}\right) .
$$

$\varphi$ reflects the trend of convergence in the numerical solution. An acute angle indicates better convergence, and an obtuse angle indicates oscillation. Therefore, when $\varphi$ is acute, $\lambda_{k}$ should be increased appropriately; when $\varphi$ is obtuse, $\lambda_{k}$ should be decreased appropriately. After many preliminary calculations, accurate results are calculated as follows:

$$
\lambda_{k}=\left\{\begin{array}{lr}
\sqrt{2} \lambda_{k-1}, & \varphi<\pi / 4 \\
\lambda_{k-1}, & \pi / 4 \leq \varphi<\pi / 2 \\
\lambda_{k-1} / \sqrt{2}, & \varphi \geq \pi / 2
\end{array}\right.
$$

For each time step, the adaptive relaxed PI (ARPI) can be summarized as follows:

(1) Set an initial $\mathbf{h}_{0}$ and tolerance $\varepsilon$, and let $k=1$.

(2) Solve Eq. (11) and calculate the current iteration increment $\Delta \mathbf{h}$.

(3) Calculate the $k$ th relaxation factor $\lambda_{k}$ : If $k=1$, then $\lambda_{k}=1$; otherwise, the previous iteration increment $\Delta \overline{\mathbf{h}}$ and relaxation factor $\lambda_{k-1}$ are read, and $\lambda_{k}$ is calculated according to Eqs. (15) and (16).

(4) The calculation result of the current iteration step is corrected using Eq. (14).

(5) If $\mathbf{h}^{k+1}$ converges according to Eq. (13), then stop; otherwise, go to step 2. 
Furthermore, a multistep preconditioner strategy considering the symmetric GaussSeidel (SGS) preconditioner is used to further improve ARPI. The SGS preconditioner $\mathbf{M}_{S G S}$ is given by:

$$
\mathbf{M}_{S G S}=(\mathbf{D}-\mathbf{L}) \mathbf{D}^{-1}(\mathbf{D}-\mathbf{U}),
$$

where $\mathbf{D}$ represents the diagonal matrix of $\mathbf{A} ; \mathbf{L}$ and $\mathbf{U}$ represent the lower and upper triangular matrices of $\mathbf{A}$, respectively. The adaptive relaxed PI scheme with multistep preconditioner (MP $(m)$-ARPI) proceeds according to the following algorithm.

\section{Algorithm MP(m)-ARPI.}

Given the number of steps $m>0$, the coefficient matrix $\mathbf{A}$, and the filter parameter $\tau:$

Let $\mathbf{A}_{1}=\mathbf{A}$

For $(i=1 ; i \leq m ; i++)$

Compute an SGS preconditioner $\mathbf{M}_{S G S}^{i}$ using $\mathbf{A}_{i}$

Compute $\quad \mathbf{A}_{i+1}=\mathbf{M}_{S G S}^{i} \mathbf{A}_{i}$

End

Drop small entries of $\mathbf{M}_{S G S}^{m}$ with respect to $\tau$

$\prod_{i=1}^{m} \mathbf{M}_{S G S}^{i}$ is the preconditioner for Eqs. (11) and (12), given by:

$\prod_{i=1}^{m} \mathbf{M}_{S G S}^{i}\left(\mathbf{h}^{j+1, k}\right) \mathbf{A}\left(\mathbf{h}^{j+1, k}\right) \mathbf{h}^{j+1, k+1}=\prod_{i=1}^{m} \mathbf{M}_{S G S}^{i}\left(\mathbf{h}^{j+1, k}\right) \mathbf{f}\left(\mathbf{h}^{j+1, k}, \mathbf{h}^{j}\right)$

Solve the preconditioned system of linear equations using ARPI.

In this study, $\tau$ is set to $10^{-4}$. It is obvious that, when $m=0$, MP( 0$)$-ARPI completely degenerates to the ARPI scheme. A brief flowchart of $\mathrm{MP}(m)$-ARPI for simulating unsaturated flow is shown in Fig. 2. To verify the calculation efficiency, the speed-up 
ratio is defined as:

$$
S_{P I / M P(m)-A R P I}=\frac{T_{P I}}{T_{M P(m)-A R P I}},
$$

where $T_{P I}$ and $T_{M P(m)-A R P I}$ are the runtimes of PI and MP(m)-ARPI, respectively. Based on the finite volume format of the RE and the improved PI scheme, the program for simulating unsaturated flow was independently developed in MATLAB version R2014a and executed on a PC with an i5-10400F CPU.

\section{Numerical Results}

\subsection{Example 1 (homogeneous soil)}

To verify the effectiveness of the proposed method, we present an example in which a 1D steady-state, transient unsaturated flow is simulated in homogeneous porous media. The mathematical model is shown in Fig. 1, where the soil thickness $(L)$ is 10 $\mathrm{m}$; the other parameters are $\alpha=8 \times 10^{-4}, \theta_{s}=0.35, \theta_{r}=0.14$, and $K_{s}=1 \times 10^{-4} \mathrm{~m} / \mathrm{s}$. The initial condition is assumed to be $h(z)=0$. The boundary conditions are set as:

$$
\begin{gathered}
h(z=0)=h_{d}, \\
h(z=L)=0,
\end{gathered}
$$

where $h_{d}$ represents the pressure head of dry soil, defined as $h_{d}=-10^{3} \mathrm{~m}$. An analytical solution for steady-state, transient unsaturated flow is given by (Tracy, 2006):

$$
\begin{gathered}
h_{\text {ana-steady }}(z)=\frac{1}{\alpha} \log \left(\left(1-e^{\alpha h_{d}}\right) e^{\frac{\alpha}{2}(L-z)}\left[\frac{\sinh (\alpha z / 2)}{\sinh (\alpha L / 2)}\right]+e^{\alpha h_{d}}\right), \\
h_{\text {ana-transient }}(z, t)=\frac{1}{\alpha} \log \left(h_{t}^{*}(z, t)+h_{s}^{*}(z)+e^{\alpha h_{d}}\right),
\end{gathered}
$$

where:

$$
h_{s}^{*}(z)=\left(1-e^{\alpha h_{d}}\right) \times\left(1-e^{-\alpha z}\right) /\left(1-e^{-\alpha L}\right),
$$




$$
h_{t}^{*}(z, t)=\frac{2\left(1-e^{\alpha h_{d}}\right)}{L c} e^{\alpha(L-z) / 2} \sum_{k=1}^{\infty}(-1)^{k}\left(\frac{\lambda_{k}}{\mu_{k}}\right) \sin \left(\lambda_{k} z\right) e^{-\mu_{k} t},
$$

with $c=\alpha\left(\theta_{s}-\theta_{r}\right) / K_{s}, \lambda_{k}=k \pi / L$, and $\mu_{k}=\left(\alpha^{2} / 4+\lambda_{k}^{2}\right) / c$.

Figure 3 shows the condition number with and without using the MP algorithm for different numbers of nodes in solving steady-state flow problems. Clearly, the condition number increases as the number of nodes increases, and the condition number can be greatly reduced using the MP algorithm, particularly MP(4). Moreover, Fig. 4 reveals that the convergence rate of PI with a constant relaxation coefficient (CPI) is slightly faster than that of PI. The performance of $\mathrm{MP}(m)$-ARPI is faster than that of $\mathrm{MP}(m)-\mathrm{PI}$, and becomes faster as $m$ increases. However, Fig. 5 indicates that the speed-up ratio relative to PI does not increase monotonically. The maximum speed-up ratio occurs when $m=3$ for $\operatorname{MP}(m)$-PI and $\operatorname{MP}(m)$-ARPI. It can also be seen that the speed-up ratio of MP $(m)$-ARPI relative to PI increases as the number of nodes increases. As shown in Figs. 6(b) and 6(c), PI and ARPI are affected by the initial conditions, and the maximum relative error is unstable. However, the maximum relative error of MP(3)-ARPI under different initial conditions decreases as the number of nodes increases (Fig. 6(a)).

For the transient flow problem, the total simulation time is $5 \mathrm{~h}$. For different iteration methods, Table 1 summarizes the average number of iterations and runtimes under different conditions. The average number of iterations increases as the number of nodes increases, and decreases as the time step decreases. MP(3)-ARPI consistently requires the fewest iterations. The numerical results for the runtimes show that MP(2)-ARPI and MP(3)-ARPI offer significant acceleration compared to PI and ARPI. Compared with MP(2)-ARPI, MP(3)-ARPI achieves a better acceleration effect when there are a larger 
number of nodes. The numerical solutions obtained by MP(3)-ARPI are highly consistent with the analytical solutions (Fig. 7).

The test results indicate that the proposed $\mathrm{MP}(m)$-ARPI effectively simulates $1 \mathrm{D}$ steady-state and transient unsaturated flow in homogeneous porous media. The results illustrate that $\operatorname{MP}(m)$-ARPI should use $m=2$ or 3 to ensure computational efficiency. When the system of linear equations is more ill-conditioned, MP(3)-ARPI is optimal.

\subsection{Example 2 (two-layer soils)}

In two-layer soils, the coefficient matrix is extremely ill-conditioned because of the different physical parameters between soil layers (Liu et al., 2015; Zhu et al., 2019). To further verify the effectiveness of the proposed scheme, this example simulates the 1D unsaturated flow in a two-layer soil. The mathematical model is shown in Fig. 8, where $L_{1}=L_{2}=5 \mathrm{~m}$. The number of nodes $N$ in each layer of soil is set to 80 . The parameters and boundary conditions are consistent with those in Example 1. Table 2 presents various parameters for this example, with the hydraulic conductivity for layer 1 set to $10^{-1} \mathrm{~m} / \mathrm{s}$ and that for layer 2 ranging from $10^{-2}-10^{-9} \mathrm{~m} / \mathrm{s}$.

Figure 9 shows that the condition number of the coefficient matrix increases as the hydraulic conductivity of the two layers diverges, but the condition number can be greatly reduced using the MP algorithm. In Case 8, in particular, using MP(3) reduces the condition number by an order of $10^{7}$. The computed results for Cases 2 and 6 are depicted in Figs. 10(a) and 10(b). From Fig. 11(a), it can be seen that the proposed MP(3)-ARPI generally requires the fewest iterations. Additionally, the speed-up ratios of MP(3)-ARPI relative to PI and ARPI are significant (Fig. 11(b)). 
Similar to Example 1, the 1D transient unsaturated flow was examined in two-layer soils. The parameters of the two-layer soils are listed in Table 3. MP(3)-ARPI was used to solve this example, with a total simulation time of $25 \mathrm{~h}$ a time step of $0.05 \mathrm{~h}$, and the initial condition $h(z, t=0)=-10^{3}$. The computed results are shown in Fig. 12.

\subsection{Example 3 (2D transient flow)}

In this example, we simulate 2D transient flow in homogeneous porous media. In Fig. 13, the length $(L)$ and width $(W)$ of the model are both set to $1 \mathrm{~m}$, and the boundary conditions are constant heads. The top boundary conditions can be expressed as follows:

$$
h(x, L, t)=\frac{1}{\alpha} \log \left(\left(1-e^{\alpha h_{d}}\right) \sin \left(\frac{\pi x}{W}\right)+e^{\alpha h_{d}}\right) .
$$

The initial condition is $h(z, t=0)=h_{d}=-1 m$, and the parameters are unchanged from the previous example, except that $\alpha=2 \times 10^{-5}$. The $2 \mathrm{D}$ analytical solution $h_{\text {ana }}(x, z, t)$ can be obtained as follows (Tracy, 2006):

$$
\begin{gathered}
h_{\text {ana }}(x, z, t)=\frac{1}{\alpha} \log \left(h_{t}^{*}(x, z, t)+h_{s}^{*}(x, z, t)+e^{\alpha h_{d}}\right), \\
h_{t}^{*}(x, z, t)=\frac{2\left(1-e^{\alpha h_{d}}\right)}{L c} \sin \left(\frac{\pi x}{W}\right) e^{\alpha(L-z) / 2} \sum_{k=1}^{M}(-1)^{k}\left(\frac{\lambda_{k}}{\mu_{k}}\right) \sin \left(\lambda_{k} z\right) e^{-\mu_{k} t}, \\
h_{s}^{*}(x, z, t)=\left(1-e^{\alpha h_{d}}\right) e^{\frac{\alpha(L-z)}{2}} \sin \left(\frac{\pi x}{W}\right) \frac{\sinh (\beta z)}{\sinh (\beta L)},
\end{gathered}
$$

where $\beta=\sqrt{\alpha^{2} / 4+(\pi / W)^{2}}$.

The total simulation time and time step were set to $1 \mathrm{~h}$ and $0.05 \mathrm{~h}$, respectively. Figure 14 shows that the condition number of the coefficient matrix increases as the mesh density increases when solving the 2D transient flow problem. Similarly, the condition number can be reduced significantly by using the MP algorithm. In Fig. 15(a), the average number of iterations required by MP(3)-ARPI is the lowest under different 
mesh densities. The calculation efficiency of MP(3)-ARPI is higher than that of MP(2)ARPI when the mesh density is $30 \times 30$ (Fig. $15(\mathrm{~b})$ ). This numerical result is similar to that of Example 1. Figure 16 shows the numerical results obtained using MP(3)-ARPI. Compared with the analytical solution (Fig. 17), the accuracy of the numerical solution is of order $10^{-4}$.

\section{Summary and Conclusions}

PI is often used to solve the RE and is easier to implement than Newtonian iteration, as there is no need to compute any derivatives. However, PI has a poor convergence rate and is time-consuming. To improve the convergence rate of PI, we have presented an improved PI scheme that combines adaptive relaxation and multistep preconditioner strategies. The results demonstrate the effectiveness of the improved PI scheme. The following conclusions can be drawn:

(1) The proposed scheme effectively simulates unsaturated flow in porous media. The results show that the proposed $\operatorname{MP}(m)$-ARPI can effectively reduce the condition number of the coefficient matrix derived from the RE. Additionally, compared with conventional PI, CPI, and ARPI, the proposed MP(m)-ARPI offers faster convergence, higher computational efficiency, and enhanced robustness.

(2) The parameter $m$ in the proposed method should be set to 2 or 3 to ensure efficient calculations. Moreover, the results demonstrate that MP(3)-ARPI is optimal for simulating unsaturated flow in porous media when the condition number of the system of linear equations is large (i.e., infiltration in two-layer soils). Compared with the analytical solution, MP(3)-ARPI has a high degree of accuracy. 


\section{Acknowledgements}

This work was supported by the National Key R\&D Program of China [no. 2018YFC1504702]; and the National Natural Science Foundation of China [no. 41672282].

\section{Data availability}

All data generated or analyzed during this study are included within the article.

\section{Conflict of interest}

The authors declare that they have no conflict of interest.

\section{References}

Baron, V., Coudière, Y., Sochala, P., 2017. Adaptive multistep time discretization and linearization based on a posteriori error estimates for the Richards equation. Applied Numerical Mathematics, 112, 104-125.

Benzi, M., 2002. Preconditioning techniques for large linear systems: a survey. Journal of Computational Physics, 182(2), 418-477.

Brenner, K., Cancès, C., 2017. Improving Newton's Method Performance by Parametrization: The Case of the Richards Equation. SIAM Journal on Numerical Analysis, 55(4), 1760-1785.

Celia, M., Bouloutas, E., Zarba, R., 1990. A general mass-conservative numerical solution for the unsaturated flow equation. Water Resources Research. 26, 14831496.

Chávez-Negrete, C., F.J. Domínguez-Mota, D. Santana-Quinteros, 2018. Numerical solution of Richards equation of water flow by generalized finite differences. Computers and Geotechnics, 101, 168-175. 
De Smedt, B., Pattyn, F., De Groen, P. 2010. Using the unstable manifold correction in a Picard iteration to solve the velocity field in higher-order ice-flow models. Journal of Glaciology, 56(196), 257-261.

Dolejší, V., Kuraz, M., Solin, P., 2019. Adaptive Higher-Order Space-Time Discontinuous Galerkin Method for the Computer Simulation of VariablySaturated Porous Media Flows. Applied Mathematical Modelling, 72, 276-305.

Duc, N.C., Nam, M.D., Antille, D.L.; van Genuchten, M.T. 2020. A control volume scheme using compact integrated radial basis function stencils for solving the Richards equation. Journal of Hydrology, 580, 124240.

Durbin, T., Delemos, D. 2007. Adaptive Underrelaxation of Picard Iterations in Ground Water Models. Ground Water, 45(5), 648-651.

Gardner, W. R., 1958. Some steady-state solutions of the unsaturated moisture flow equation with application to evaporation from a water table. Soil Science, 85(4), 228-232.

Illiano, D., Pop, I. S., Radu, F. A. 2020. Iterative schemes for surfactant transport in porous media. Computational Geosciences. https://doi.org/10.1007/s10596-02009949-2.

Ku, C. Y., Liu, C.Y., Su, Y., Xiao, J.E., Huang, C.C. 2017. Transient modeling of regional rainfall-triggered shallow landslides. Environmental Earth Sciences, $76(16), 570$. 
Kuraz, M., Mayer, P., Pech, P. 2015. Solving the nonlinear and nonstationary Richards equation with two-level adaptive domain decomposition (dd-adaptivity). Applied Mathematics and Computation, 267, 207-222.

Li, S.H.; Wu, L.Z.; Luo, X.H. 2020. A novel method for locating the critical slip surface of a soil slope. Engineering Applications of Artificial Intelligence, 94, 103733.

List, F., Radu, F.A., 2016. A study on iterative methods for solving Richards' equation. Computational Geosciences, 20(2), 341-353.

Liu, C.Y., C.Y. Ku, C.C. Huang, 2015. Numerical Solutions for Groundwater Flow in Unsaturated Layered Soil with Extreme Physical Property Contrasts. International Journal of Nonlinear Sciences \& Numerical Simulation, 16(7), 325-335.

Lott, P. A., Walker, H. F., Woodward, C. S., Yang, U. M., 2012. An accelerated Picard method for nonlinear systems related to variably saturated flow. Advances in Water Resources, 38, 92-101.

Morway, E.D., Niswonger, R.G., Langevin, C.D., Bailey, R.T., Healy, R.W., 2013. Modeling variably saturated multispecies reactive groundwater solute transport with MODFLOW-UZF and MT3DMS. Groundwater, 51(2), 237-251.

Paniconi, C., M. Putti, 1994. A comparison of Picard and newton iteration in the numerical solution of multidimensional variably saturated flow problems. Water Resources Research, 30, 3357-3374.

Patankar, S. V., 1980. Numerical Heat Transfer and Fluid Flow, CRC press.

Richards, L A., 1931. Capillary Conduction of Liquids Through Porous Mediums. Physics, 1(5), 318-333. 
Šimůnek, J., Šejna, M., Saito, H., Sakai, M., van Genuchten, M.T., 2009. The HYDRUS-1D Software Package for Simulating the One-Dimensional Movement of Water, Heat, and Multiple Solutes in Variably-Saturated Media. Department of Environmental Sciences, University of California Riverside, Riverside, CA.

Solin, P., Kuraz, M., 2011. Solving the nonstationary Richards equation with adaptive hp-FEM. Advances in Water Resources, 34(9), 1062-1081.

Srivastava, R., Yeh, T.C.J. 1991. Analytical solutions for one-dimensional, transient infiltration toward the water table in homogeneous layered soils. Water Resources Research. 27(5), 753-762.

Svyatskiy, D., Lipnikov, K., 2017. Second-order accurate finite volume schemes with the discrete maximum principle for solving Richards' equation on unstructured meshes. Advances in Water Resources, 104, 114-126.

Tracy, F.T., 2006. Clean two- and three-dimensional analytical solutions of Richards' equation for testing numerical solvers. Water Resources Research, 42(8), 8503.

Wang, H.F., Anderson, M.P., 1982. Introduction to groundwater modeling. Freeman.

Wang, K., Zhang, J. 2003. MSP: A Class of Parallel Multistep Successive Sparse Approximate Inverse Preconditioning Strategies. SIAM Journal on Scientific Computing, 24(4), 1141-1156.

Wang, W., Rutqvist, J., Görke, U.J., Birkholzer, J. T., Kolditz, O. 2011. Non-isothermal flow in low permeable porous media: a comparison of Richards' and two-phase flow approaches. Environmental Earth Sciences, 62(6), 1197-1207. 
Wu, L.Z., G.G. Liu, L.C. Wang, L.M. Zhang, B.E. Li, B. Li, 2016. Numerical analysis of $1 \mathrm{D}$ coupled infiltration and deformation in layered unsaturated porous medium. Environmental Earth Sciences. 75(9), 761.

Wu, L.Z., Jinsong, Huang, W. Fan, X. Li, 2020a. Hydro-mechanical coupling in unsaturated soils covering a non-deformable structure. Computers and Geotechnics. 117, 103287.

Wu, L.Z., S.R. Zhu, J.B. Peng, 2020b. Application of the Chebyshev spectral method to the simulation of groundwater flow and rainfall-induced landslides. Applied Mathematical Modelling. 80, 408-425.

Wu, L.Z., Selvadurai, A.P.S., Huang, R.Q. 2013. Two-dimensional coupled hydromechanical modeling of water infiltration into a transversely isotropic unsaturated soil region. Vadose Zone Journal. 12(4), 4949-4960.

Zambra, C.E., Dumbser, M., Toro, E.F., Moraga, N.O., 2012. A novel numerical method of high-order accuracy for flow in unsaturated porous media. International Journal for Numerical Methods in Engineering, 89, 227-240.

Zang, Y.G., Sun, D.M., Feng, P., Semprich, S., 2016. Effects of Airflow Induced by Rainfall on Shallow Groundwater Table Fluctuations. Groundwater, 55(3), 375386.

Zha, Y., Yang, J., Shi, L., Song, X., 2013. Simulating One-Dimensional Unsaturated Flow in Heterogeneous Soils with Water Content-Based Richards Equation. Vadose Zone Journal, 12(2), 1-13. 
Zha, Y., Yang, J., Yin, L., Zhang, Y., Zeng, W., Shi, L. 2017. A modified Picard iteration scheme for overcoming numerical difficulties of simulating infiltration into dry soil. Journal of Hydrology, 551, 56-69.

Zhang, Z., Wang, W., Yeh, T. J., Chen, L., Wang, Z., Duan, L., An, K., Gong, C., 2016. Finite analytic method based on mixed-form Richards' equation for simulating water flow in vadose zone. Journal of Hydrology, 537, 146-156.

Zhu, S.R., Wu, L.Z., Peng, J.B., 2020. An improved Chebyshev semi-iterative method for simulating rainfall infiltration in unsaturated soils and its application to shallow landslides, Journal of Hydrology, 590, 125157. 


\section{Figure and Table Captions}

Table 1. Numerical results for Example 1.

Table 2. Hydraulic conductivity for different cases in two-layer soils.

Table 3. Parameters of the two-layer soils.

Figure 1. Schematic drawing of control volume method based on non-uniform grid nodes and 1D infiltration model for homogeneous soil.

Figure 2. Brief flowchart of $\mathrm{MP}(m)$-ARPI.

Figure 3. Condition number with and without using MP algorithm for different numbers of nodes.

Figure 4. Comparison of convergence rates for the proposed schemes.

Figure 5. Comparison of speed-up ratio for the proposed schemes relative to PI.

Figure 6. Comparison of the maximum relative error (\%) for different numbers of nodes and initial conditions using (a) MP(3)-ARPI, (b) PI and (c) ARPI.

Figure 7. Comparison of the numerical solutions obtained using MP(3)-ARPI with analytical solutions for Example 1.

Figure 8. 1D infiltration model of two-layer unsaturated soils.

Figure 9. Condition number for Cases 1-8.

Figure 10. Numerical solutions obtained using MP(3)-ARPI: (a) Case 2; (b) Case 6.

Figure 11. Numerical results for Cases 1-8: (a) Number of iterations; (b) Speed-up ratio.

Figure 12. Numerical solution for 1D transient unsaturated flow in two-layered soils.

Figure 13 Mathematical model of a 2D transient flow.

Figure 14. Condition number for different mesh densities.

Figure 15. Numerical results for the 2D transient flow problem: (a) Average number of iterations; (b) Speed-up ratio.

Figure 16. Numerical solutions obtained using MP(3)-ARPI for 2D transient flow 
problem at (a) $0.2 \mathrm{~h}$, (b) $1 \mathrm{~h}$.

Figure 17. Absolute error of the results computed using MP(3)-ARPI relative to analytical solutions for $2 \mathrm{D}$ transient flow problem at $t=1 \mathrm{~h}$. 
Table 1. Numerical results for Example 1.

\begin{tabular}{cccccccccc}
\hline \multicolumn{2}{c}{ Conditions } & \multicolumn{4}{c}{$\begin{array}{c}\text { Average number of iterations for each } \\
\text { time step }\end{array}$} \\
\hline $\begin{array}{c}\text { Number } \\
\text { of nodes }\end{array}$ & $\Delta t$ & PI & ARPI & $\begin{array}{c}\text { MP(2)- } \\
\text { ARPI }\end{array}$ & $\begin{array}{c}\text { MP(3)- } \\
\text { ARPI }\end{array}$ & PI & ARPI & $\begin{array}{c}\text { MP(2)- } \\
\text { ARPI }\end{array}$ & $\begin{array}{c}\text { MP(3)- } \\
\text { ARPI }\end{array}$ \\
\hline \multirow{2}{*}{120} & $0.2 \mathrm{~h}$ & 399 & 245 & 15 & 11 & 15.65 & 9.82 & 2.86 & 3.04 \\
& $0.1 \mathrm{~h}$ & 287 & 180 & 14 & 11 & 22.66 & 14.70 & 5.06 & 5.57 \\
& $0.05 \mathrm{~h}$ & 207 & 127 & 13 & 10 & 32.99 & 20.98 & 9.26 & 10.49 \\
160 & $0.2 \mathrm{~h}$ & 679 & 414 & 21 & 13 & 45.83 & 28.16 & 6.13 & 6.04 \\
& $0.1 \mathrm{~h}$ & 489 & 296 & 18 & 12 & 66.07 & 40.34 & 10.57 & 10.72 \\
& $0.05 \mathrm{~h}$ & 352 & 220 & 15 & 12 & 96.09 & 60.91 & 17.98 & 19.79 \\
200 & $0.2 \mathrm{~h}$ & 1027 & 615 & 29 & 14 & 113.82 & 69.72 & 13.74 & 9.34 \\
& $0.1 \mathrm{~h}$ & 739 & 446 & 21 & 13 & 164.59 & 100.04 & 20.24 & 17.19 \\
& $0.05 \mathrm{~h}$ & 533 & 321 & 18 & 12 & 238.09 & 146.59 & 35.58 & 33.01 \\
\hline
\end{tabular}


Table 2. Hydraulic conductivity for different cases in two-layer soils.

\begin{tabular}{ccccccccc}
\hline Case number & 1 & 2 & 3 & 4 & 5 & 6 & 7 & 8 \\
\hline$K_{s 1}(\mathrm{~m} / \mathrm{s})$ & $10^{-1}$ & $10^{-1}$ & $10^{-1}$ & $10^{-1}$ & $10^{-1}$ & $10^{-1}$ & $10^{-1}$ & $10^{-1}$ \\
$K_{s 2}(\mathrm{~m} / \mathrm{s})$ & $10^{-2}$ & $10^{-3}$ & $10^{-4}$ & $10^{-5}$ & $10^{-6}$ & $10^{-7}$ & $10^{-8}$ & $10^{-9}$ \\
\hline
\end{tabular}

Table 3. Parameters of the two-layer soils.

\begin{tabular}{ccccc}
\hline Layer & $K_{s}(\mathrm{~m} / \mathrm{s})$ & $\theta_{s}$ & $\theta_{r}$ & $\alpha$ \\
\hline Soil layer 1 & $1.5162 \times 10^{-6}$ & 0.4686 & 0.106 & $1.04 \times 10^{-4}$ \\
Soil layer 2 & $6.2616 \times 10^{-5}$ & 0.3658 & 0.0286 & $2.8 \times 10^{-4}$ \\
\hline
\end{tabular}




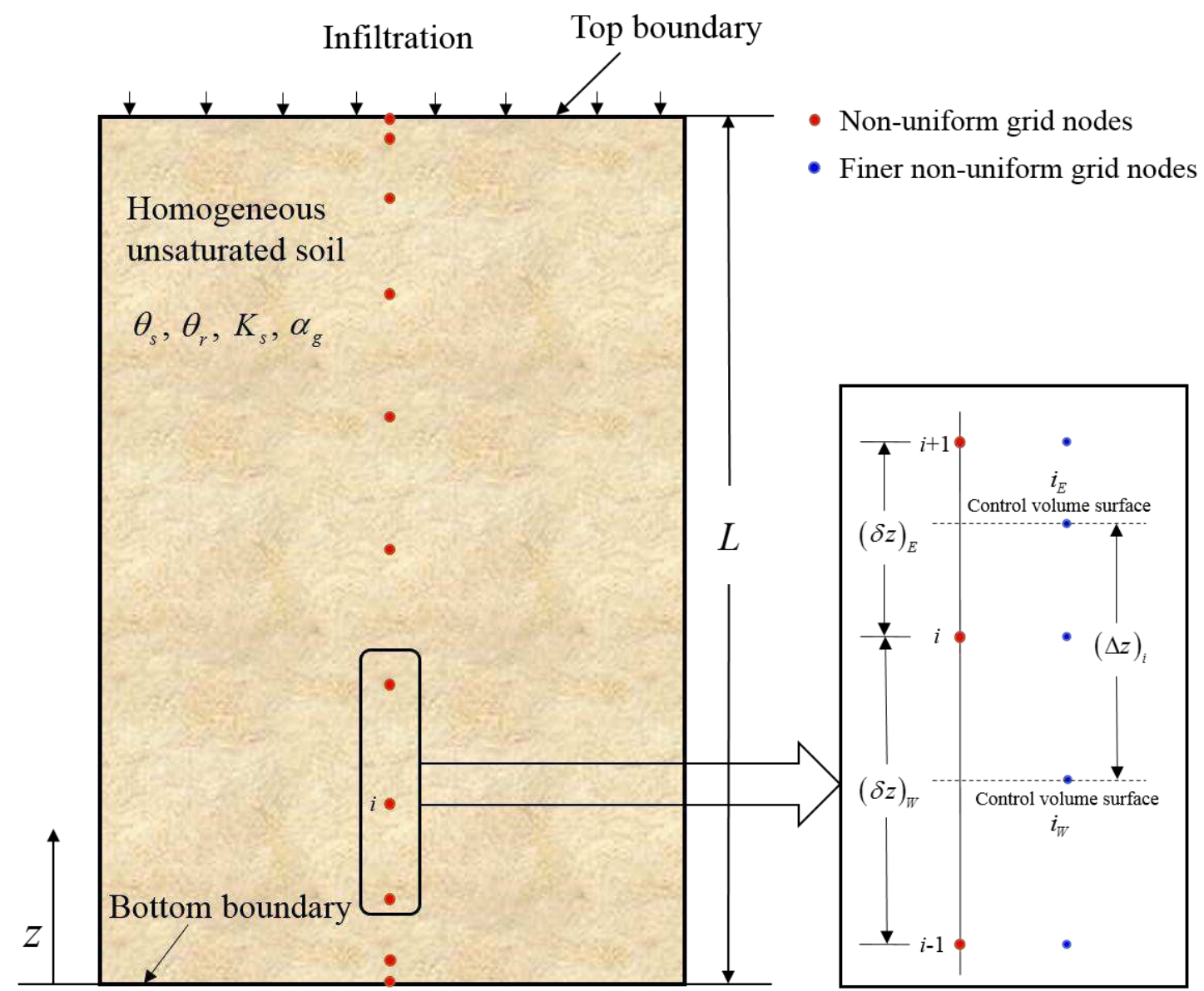

Figure 1. Schematic drawing of control volume method based on nonuniform grid nodes and 1D infiltration model for homogeneous soil. 


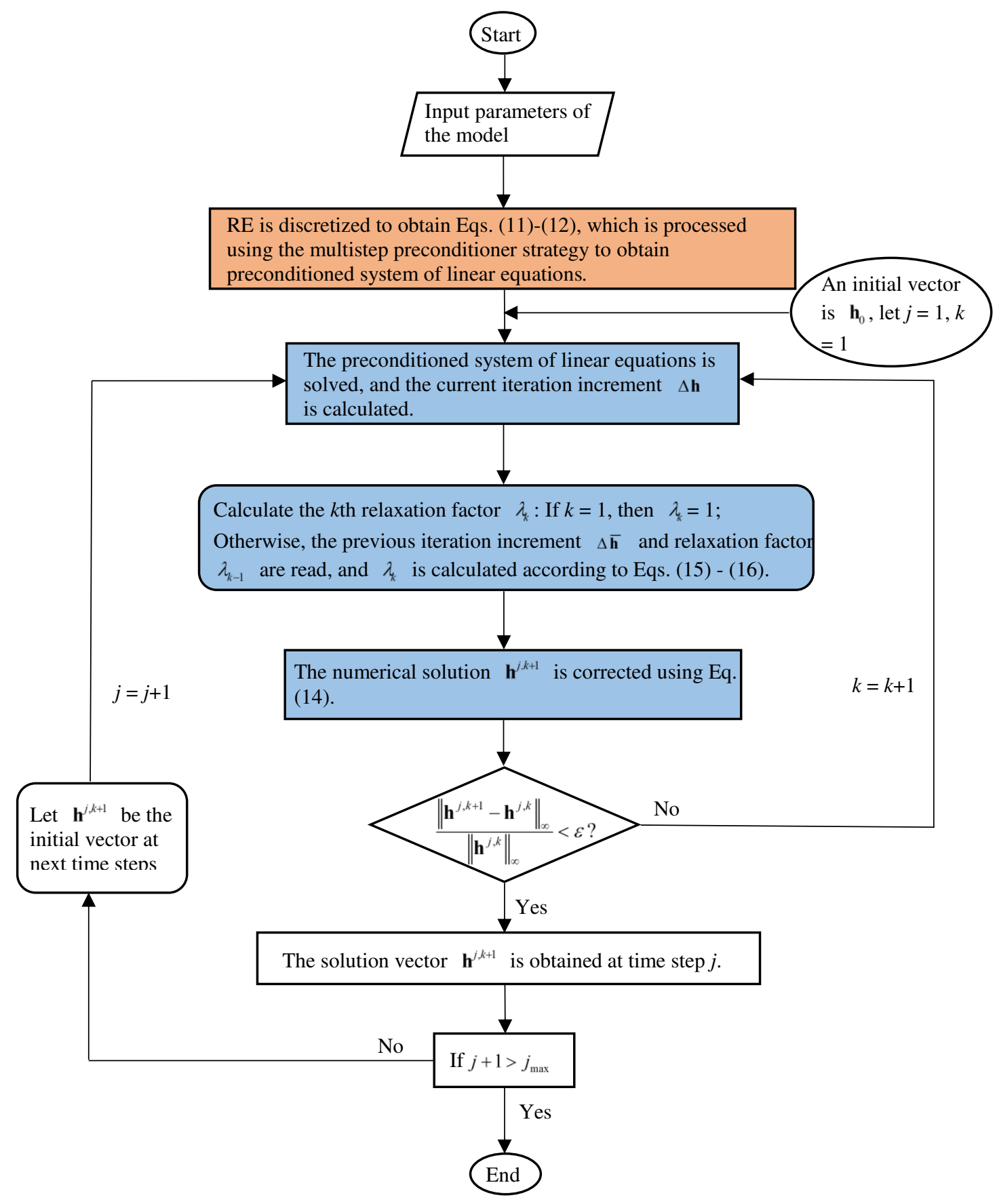

Figure 2. Brief flowchart of $\mathrm{MP}(m)$-ARPI. 


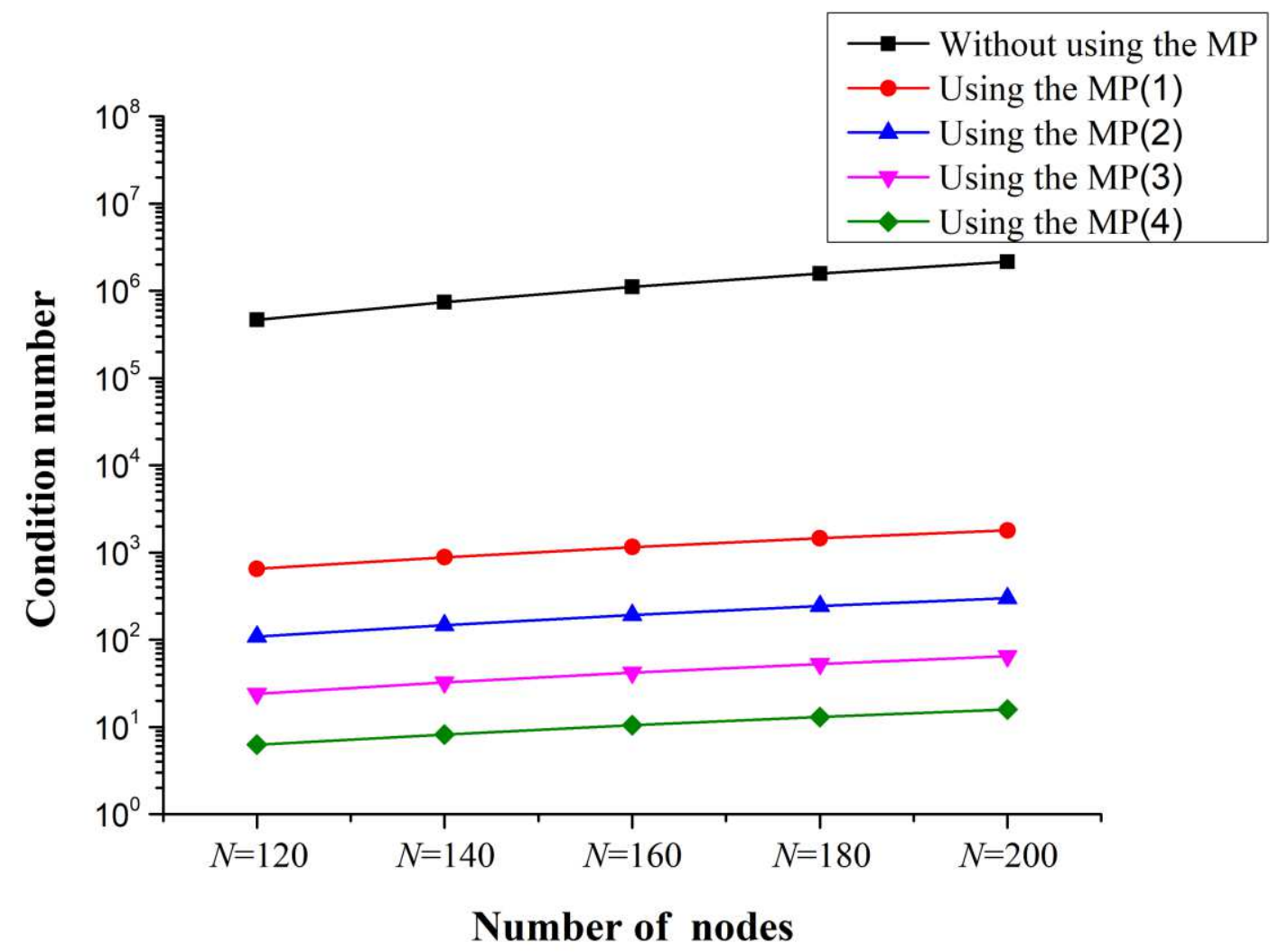

Figure 3. Condition number with and without using MP algorithm for different numbers of nodes. 


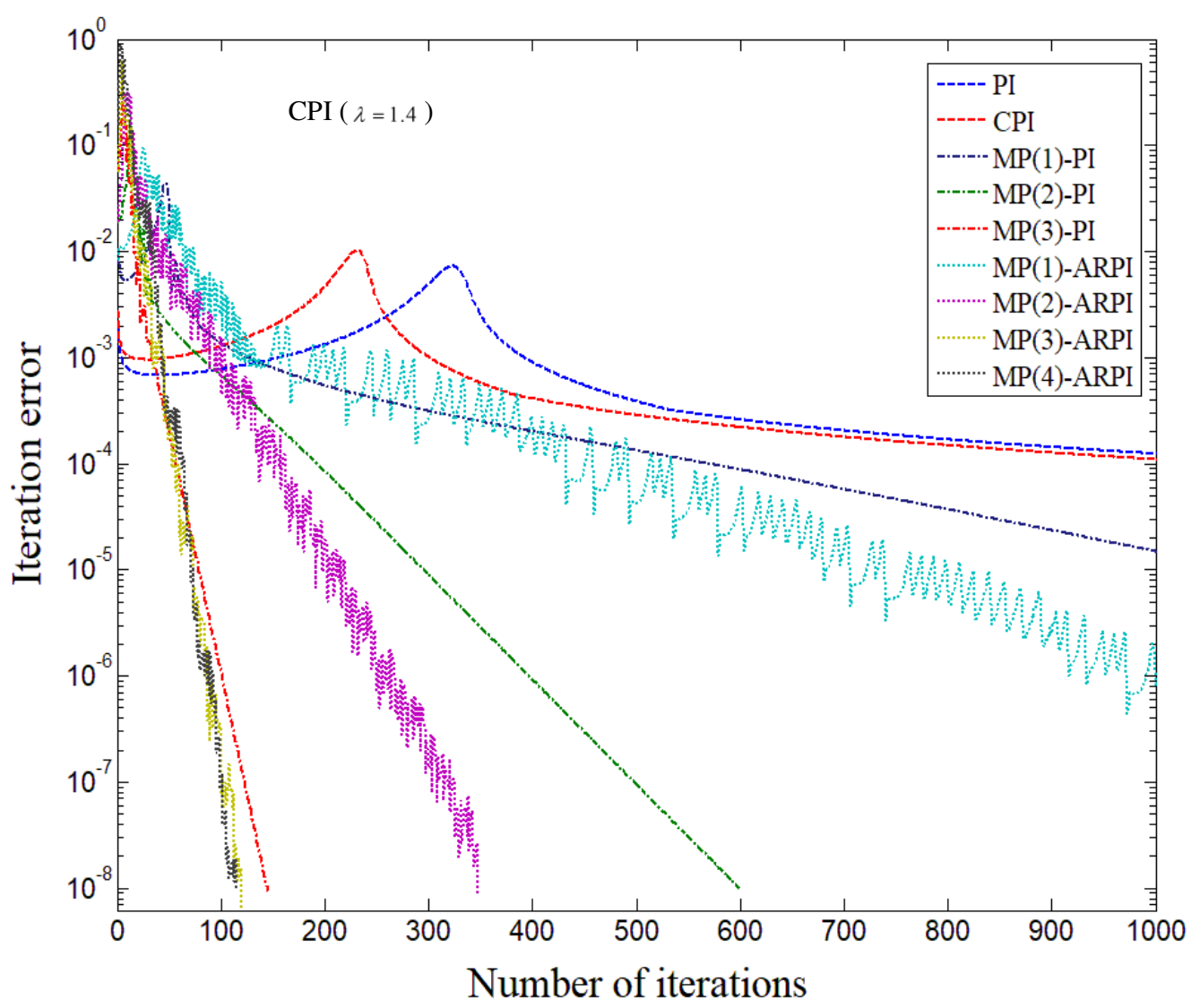

Figure 4. Comparison of convergence rates for the proposed schemes. 


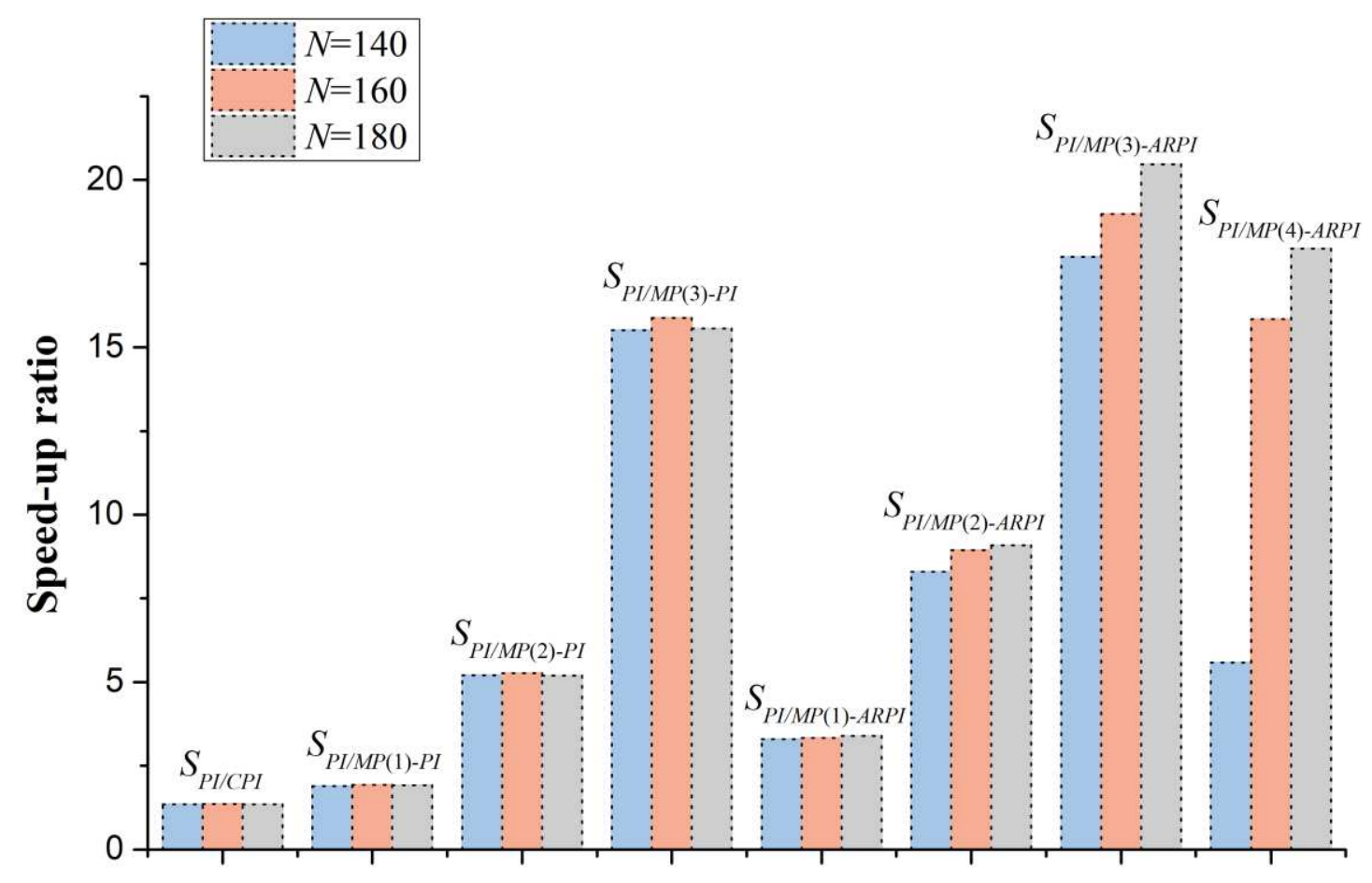

Figure 5. Comparison of speed-up ratio for the proposed schemes relative to PI. 

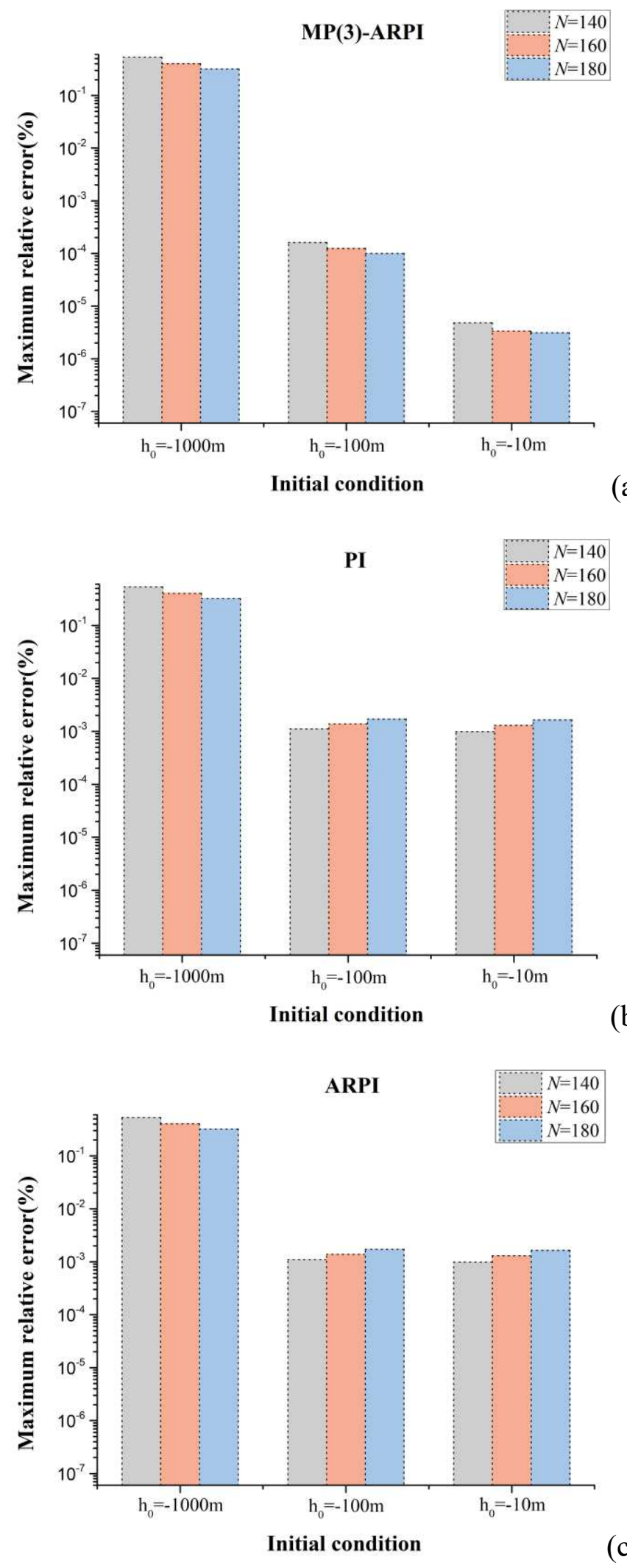

Figure 6. Comparison of the maximum relative error (\%) for different numbers of nodes and initial conditions using (a) MP(3)-ARPI, (b) PI, and (c) ARPI. 


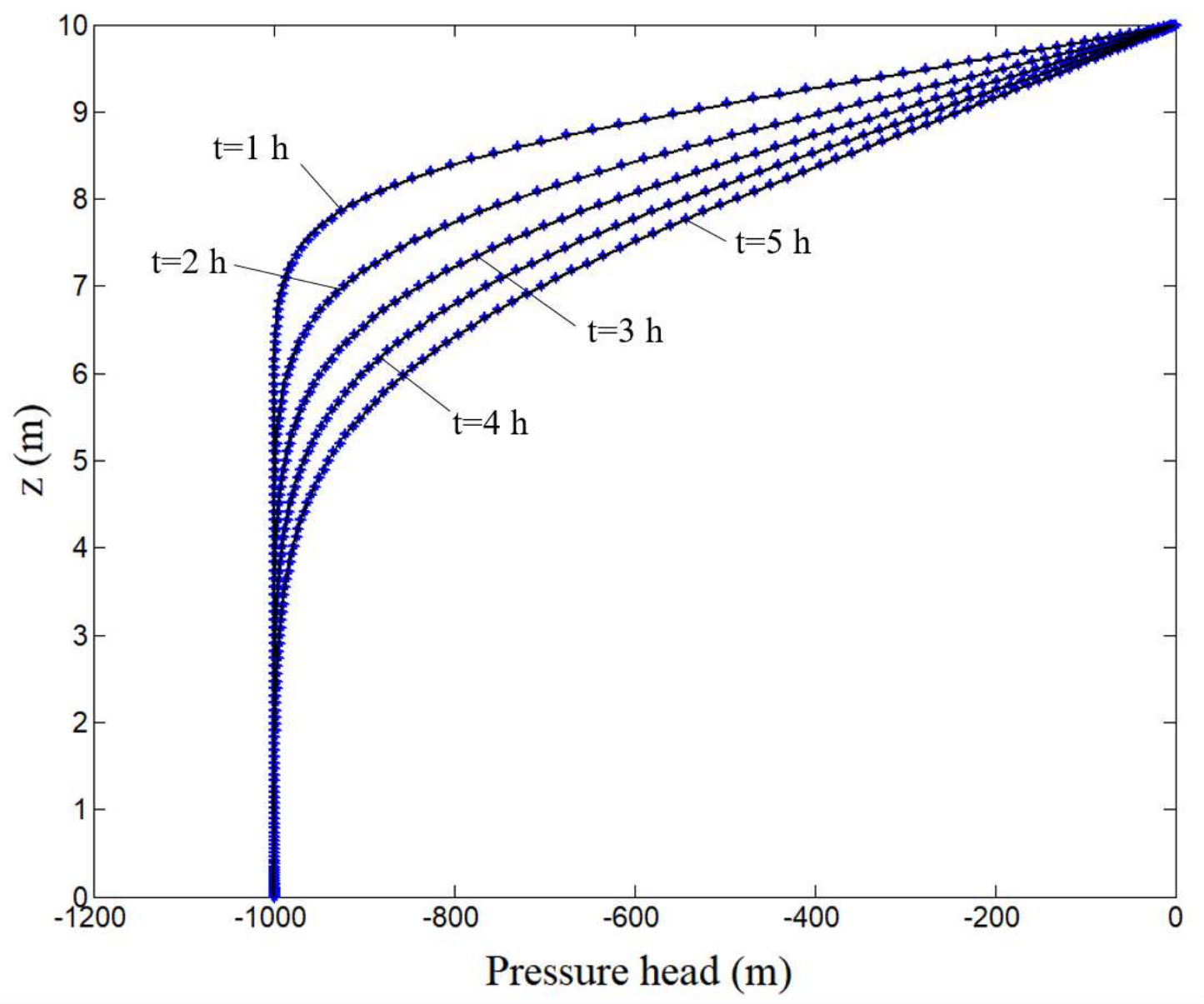

— Analytical solution (Tracy, 2006)

$* * * *$ Numerical solution by MP(3)-ARPI

Figure 7. Comparison of the numerical solutions obtained using MP(3)-ARPI with analytical solutions for Example 1. 


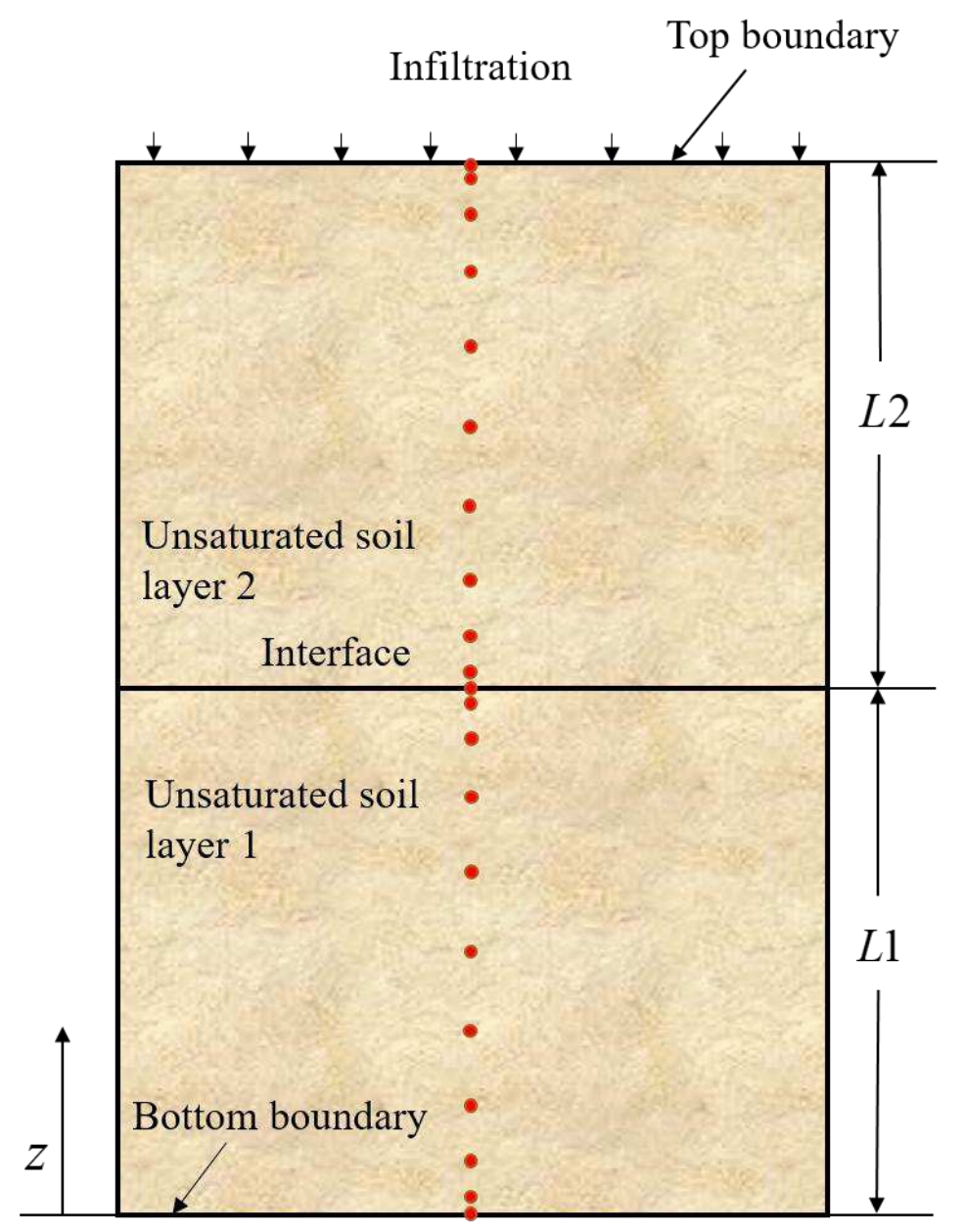

- Non-uniform grid nodes

Figure 8. 1D infiltration model of two-layer unsaturated soils. 


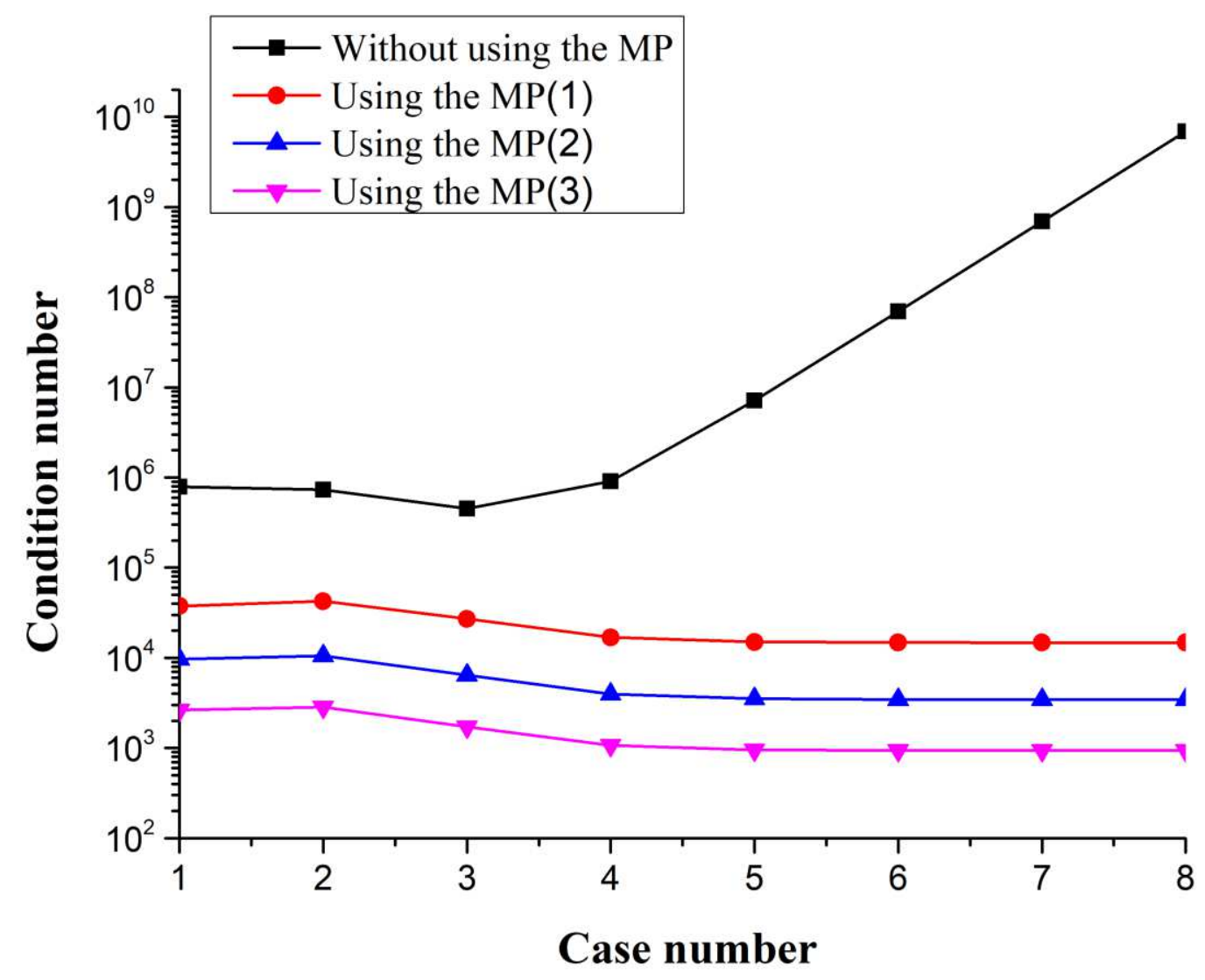

Figure 9. Condition number for Cases 1-8. 


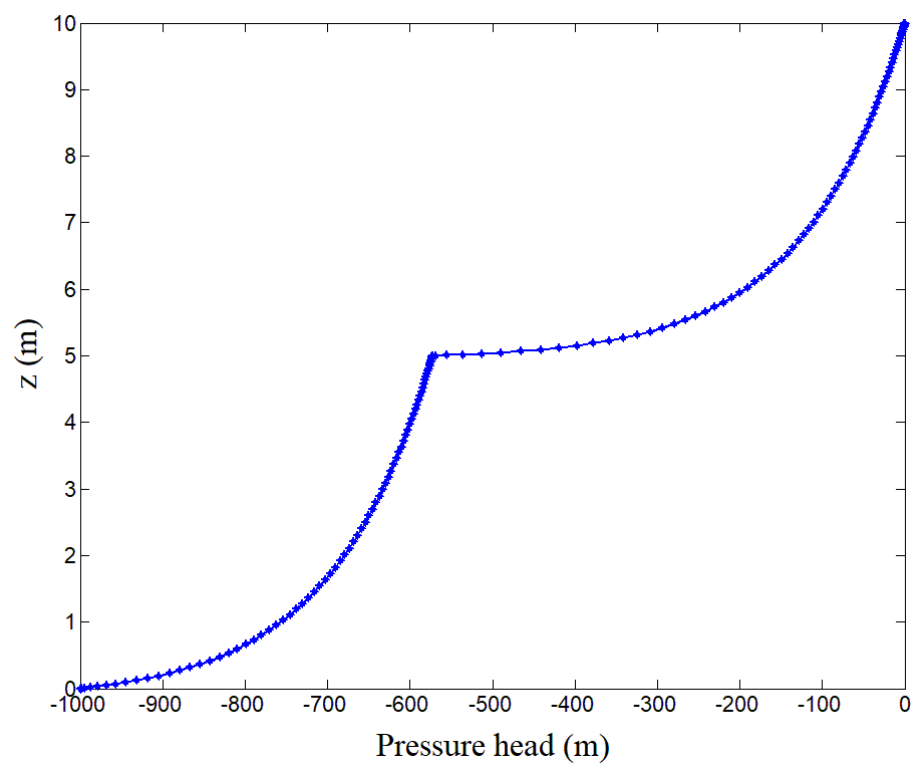

(a)

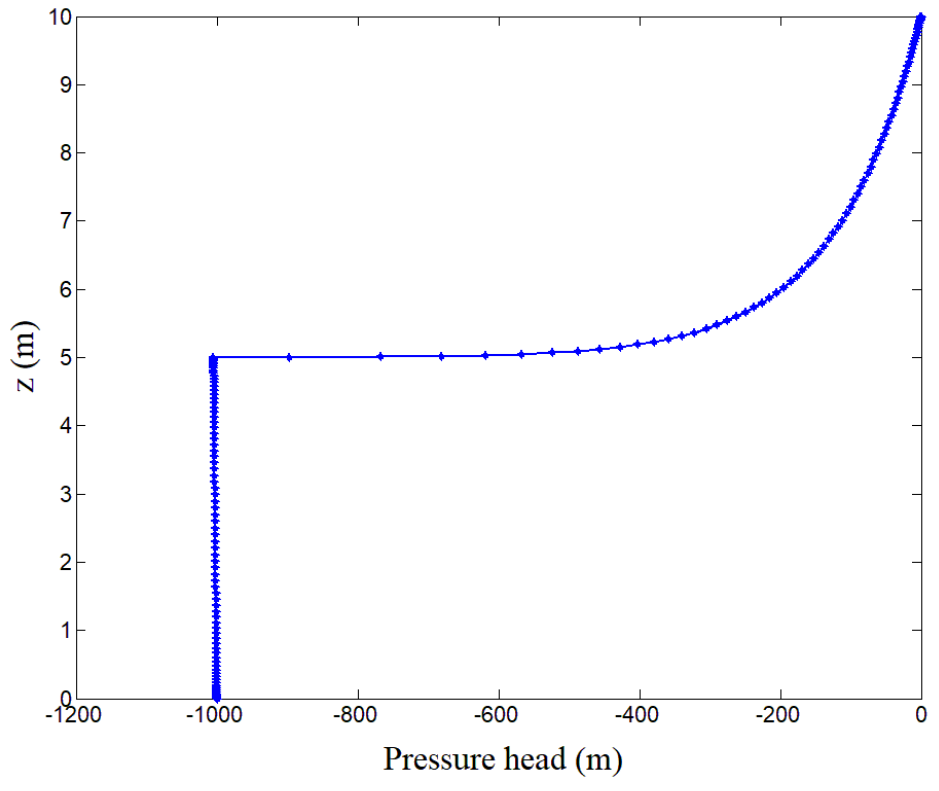

(b)

Figure 10. Numerical solutions obtained using MP(3)-ARPI: (a) Case 2; (b) Case 6. 


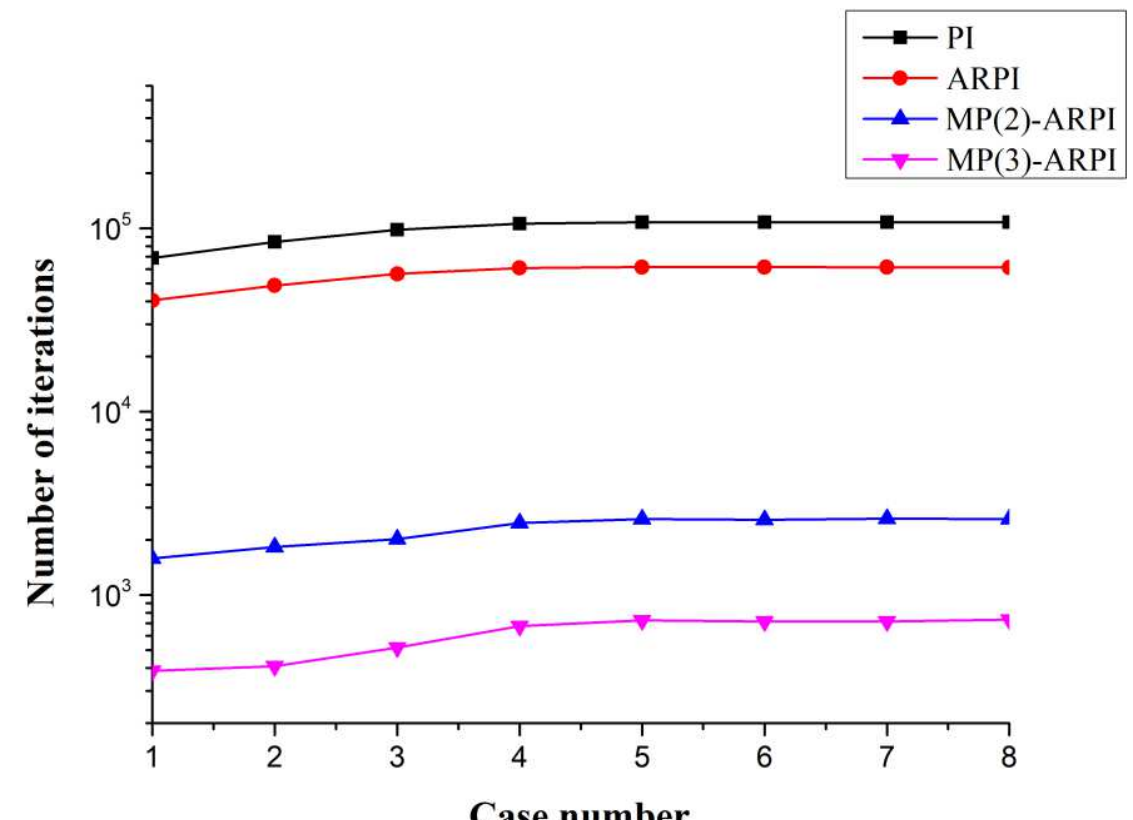

Case number

(a)

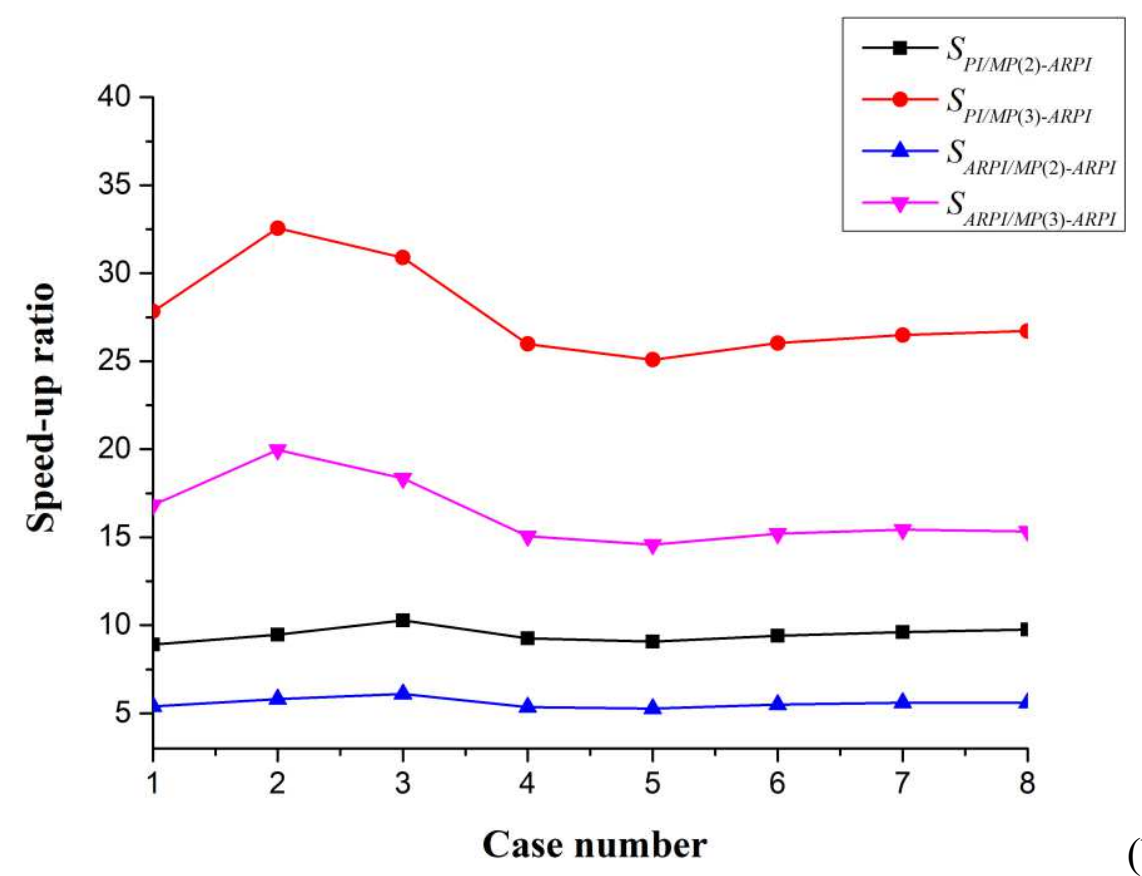

Figure 11. Numerical results for Cases 1-8: (a) Number of iterations; (b) Speed-up ratio. 


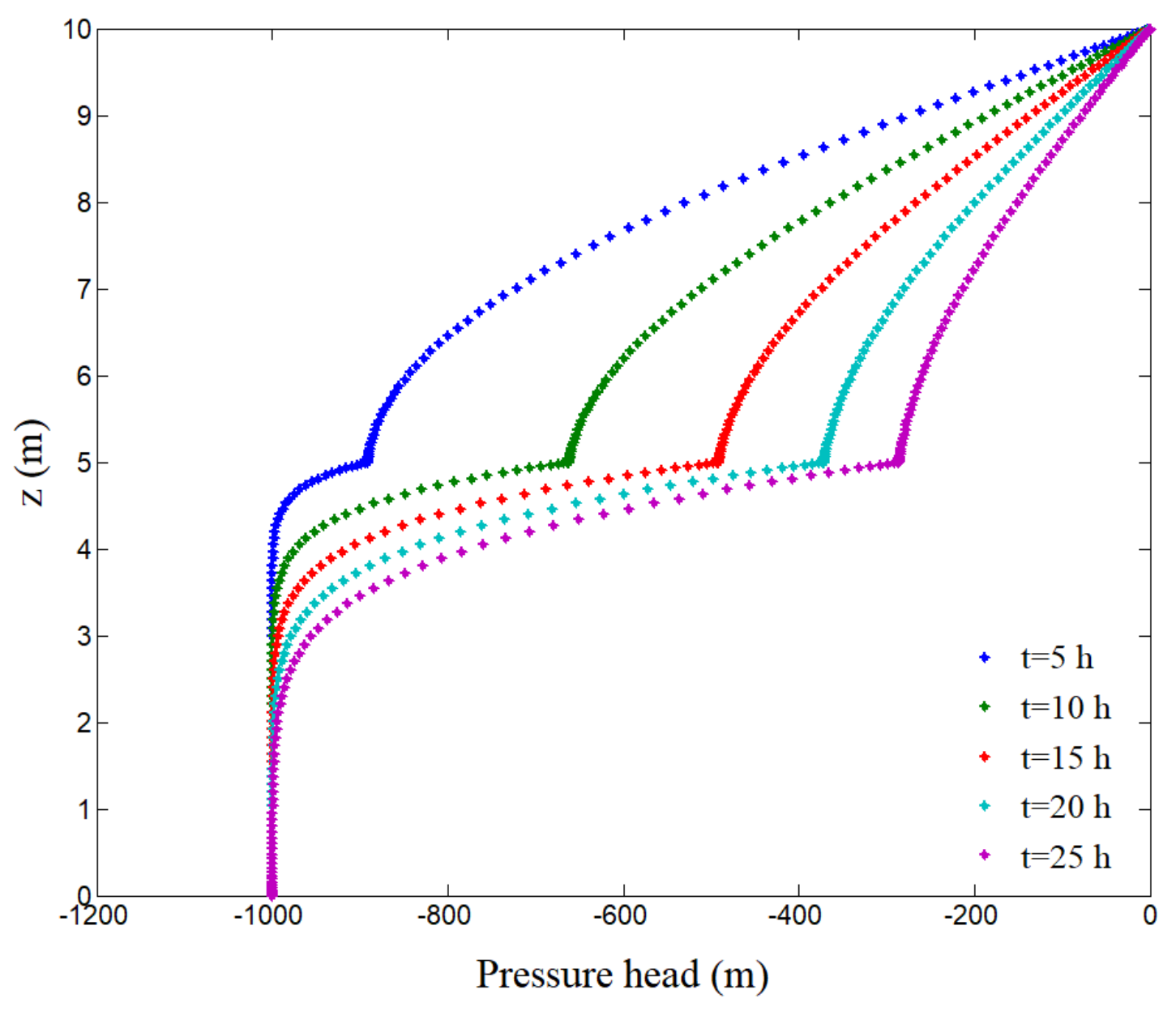

Figure 12. Numerical solution for $1 \mathrm{D}$ transient unsaturated flow in two-layered soils. 


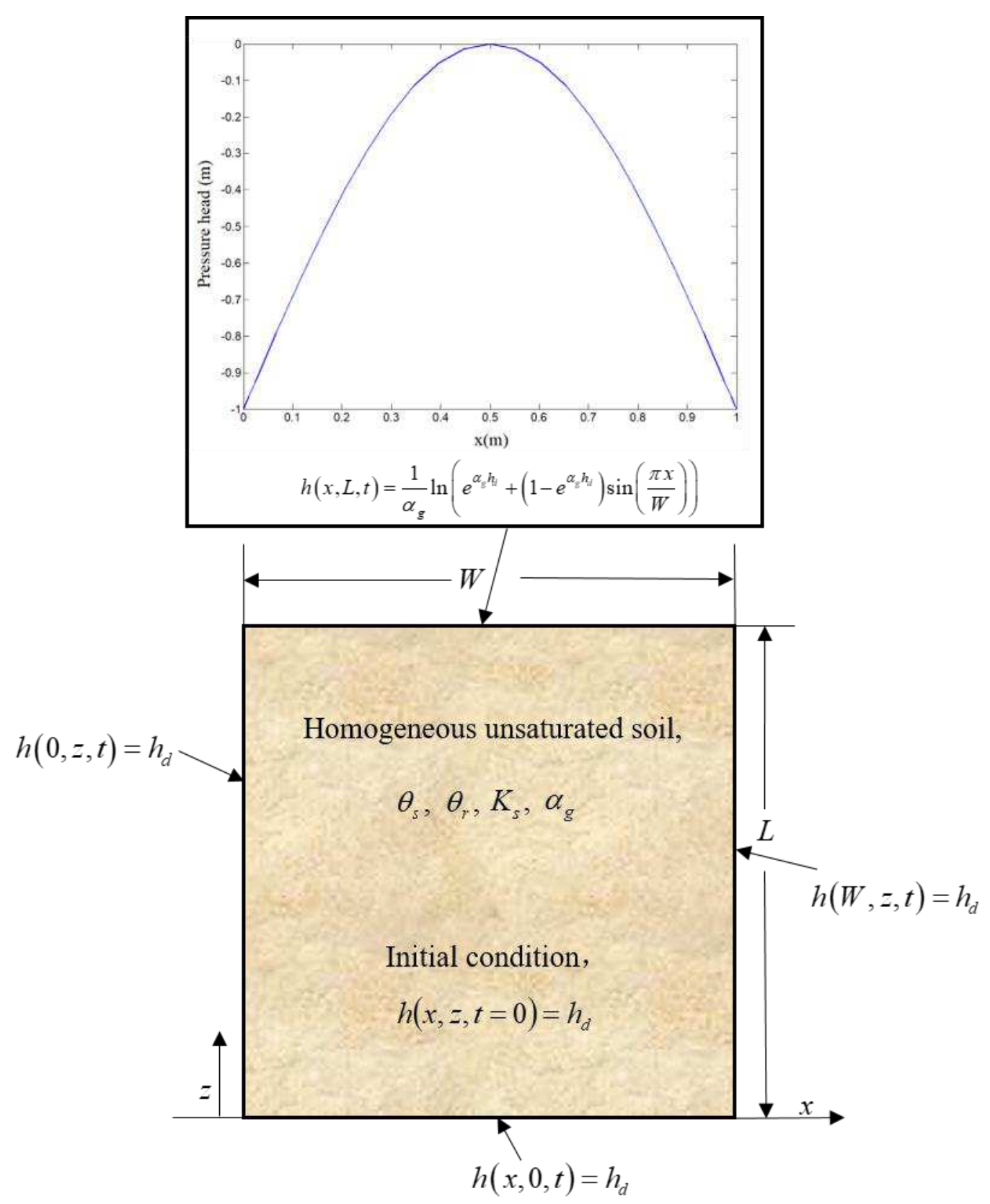

Figure 13. Mathematical model of a 2D transient flow. 


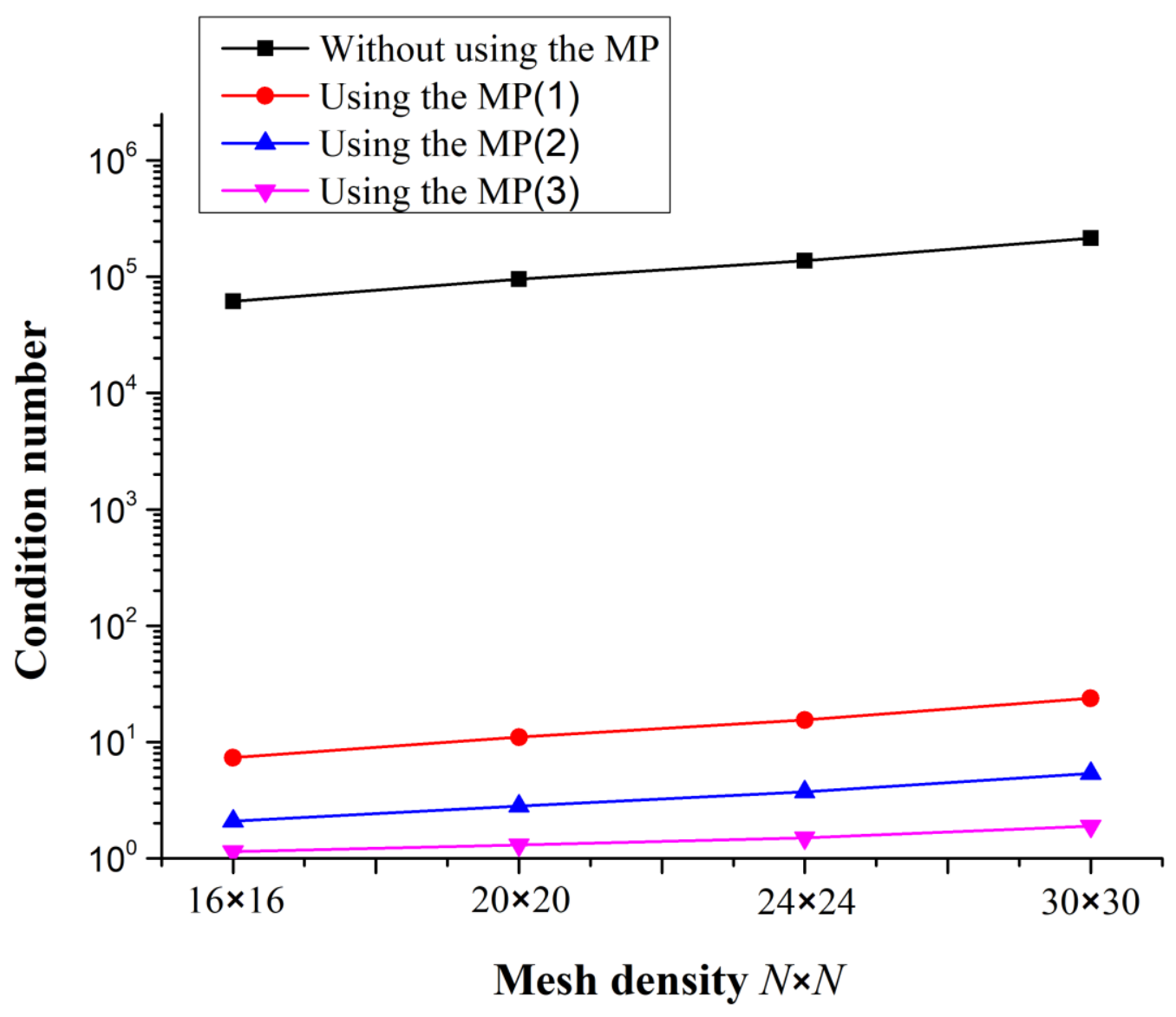

Figure 14. Condition number for different mesh densities. 


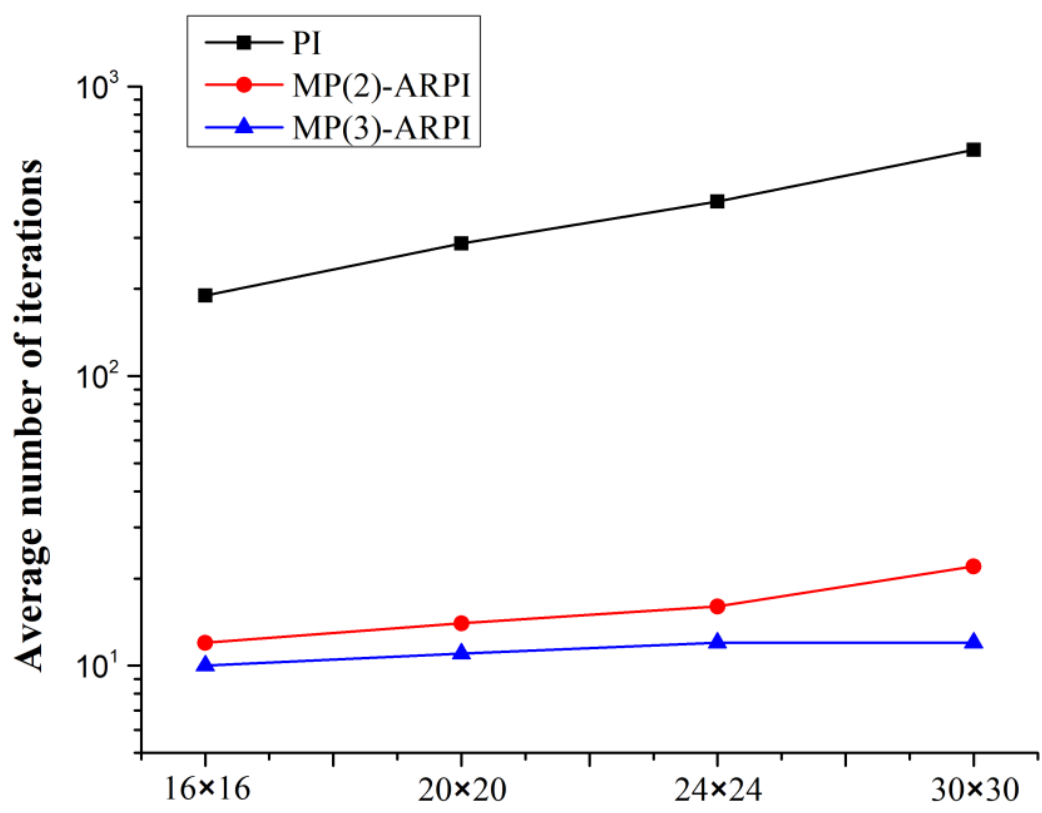

Mesh density $N \times N$

(a)

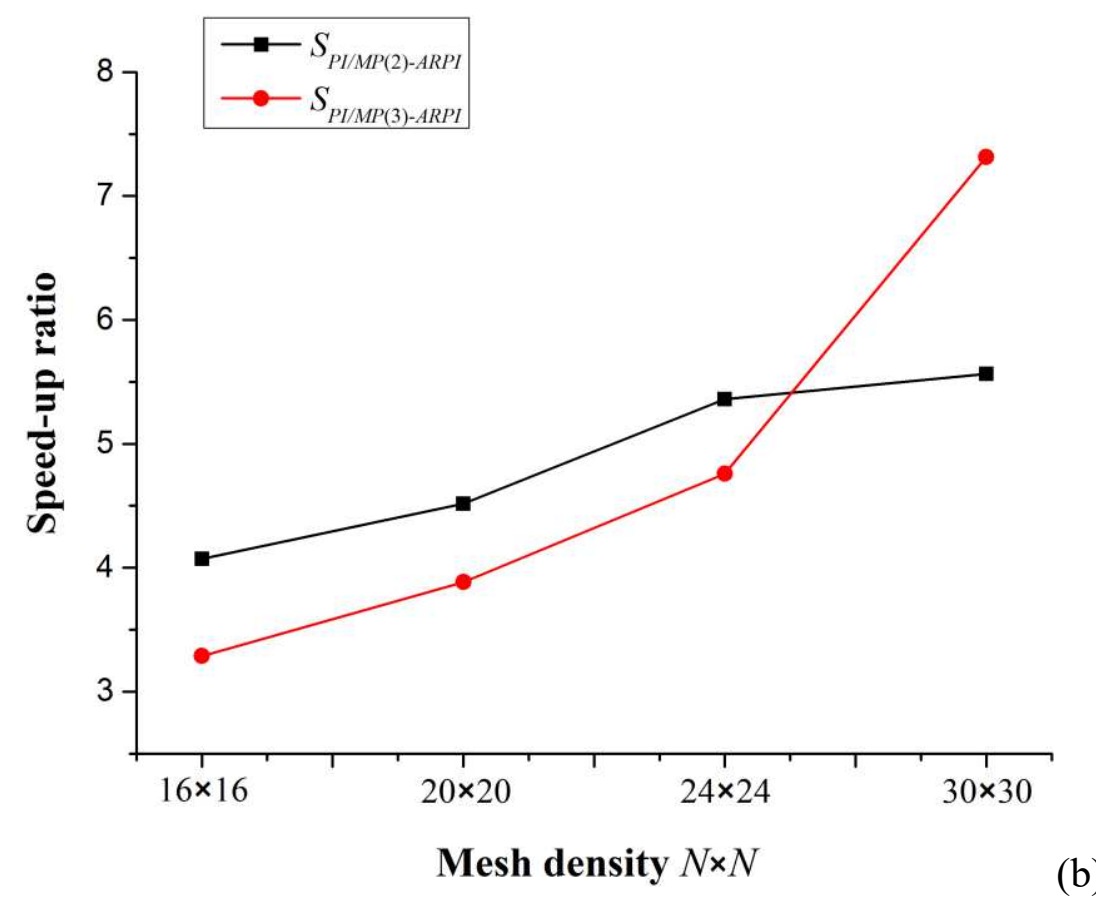

Figure 15. Numerical results for the 2D transient flow problem: (a) Average number of iterations; (b) Speed-up ratio. 

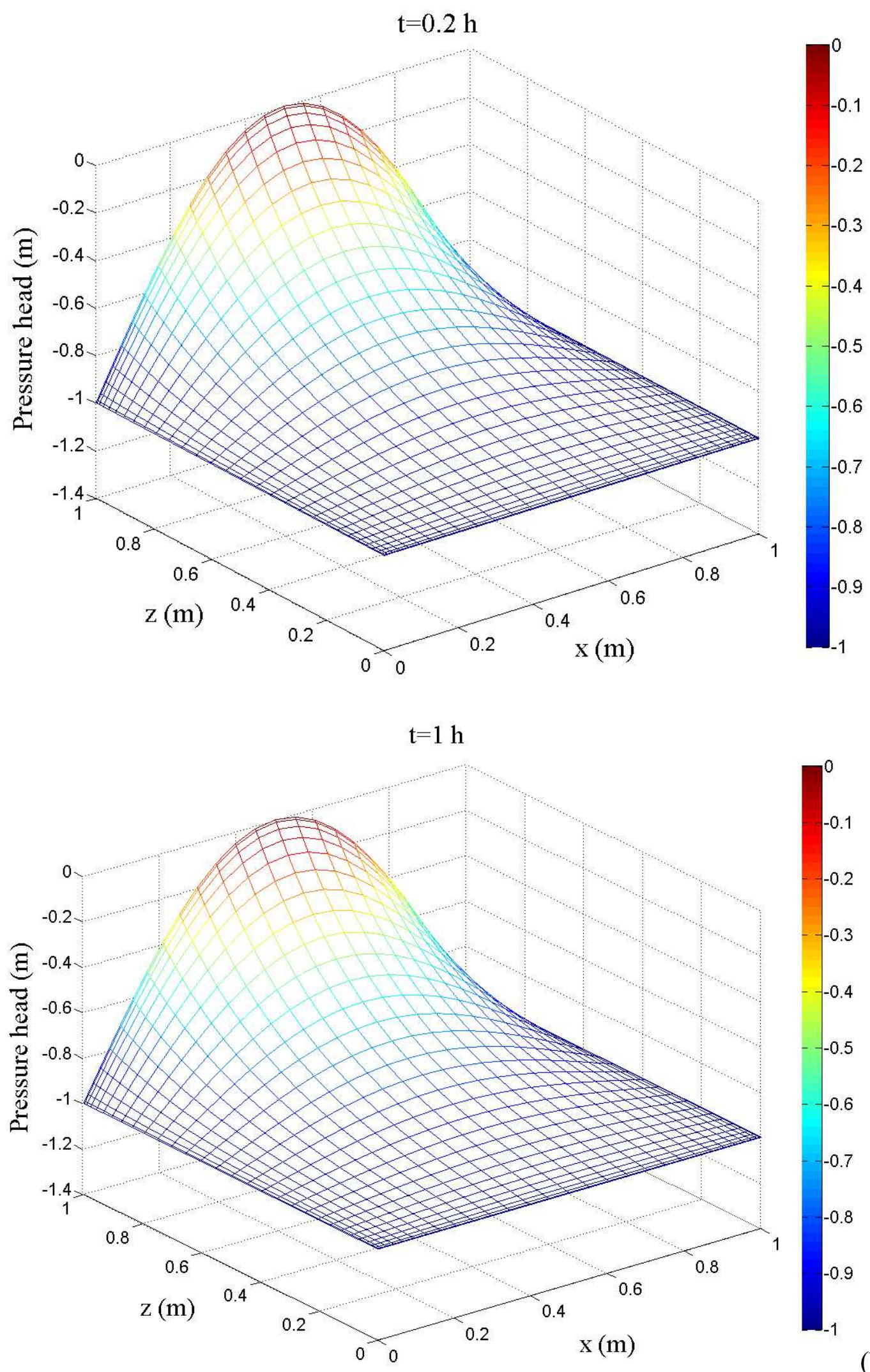

(a)

Figure 16. Numerical solutions obtained using MP(3)-ARPI for $2 \mathrm{D}$ transient flow problem at (a) $0.2 \mathrm{~h}$, (b) $1 \mathrm{~h}$. 


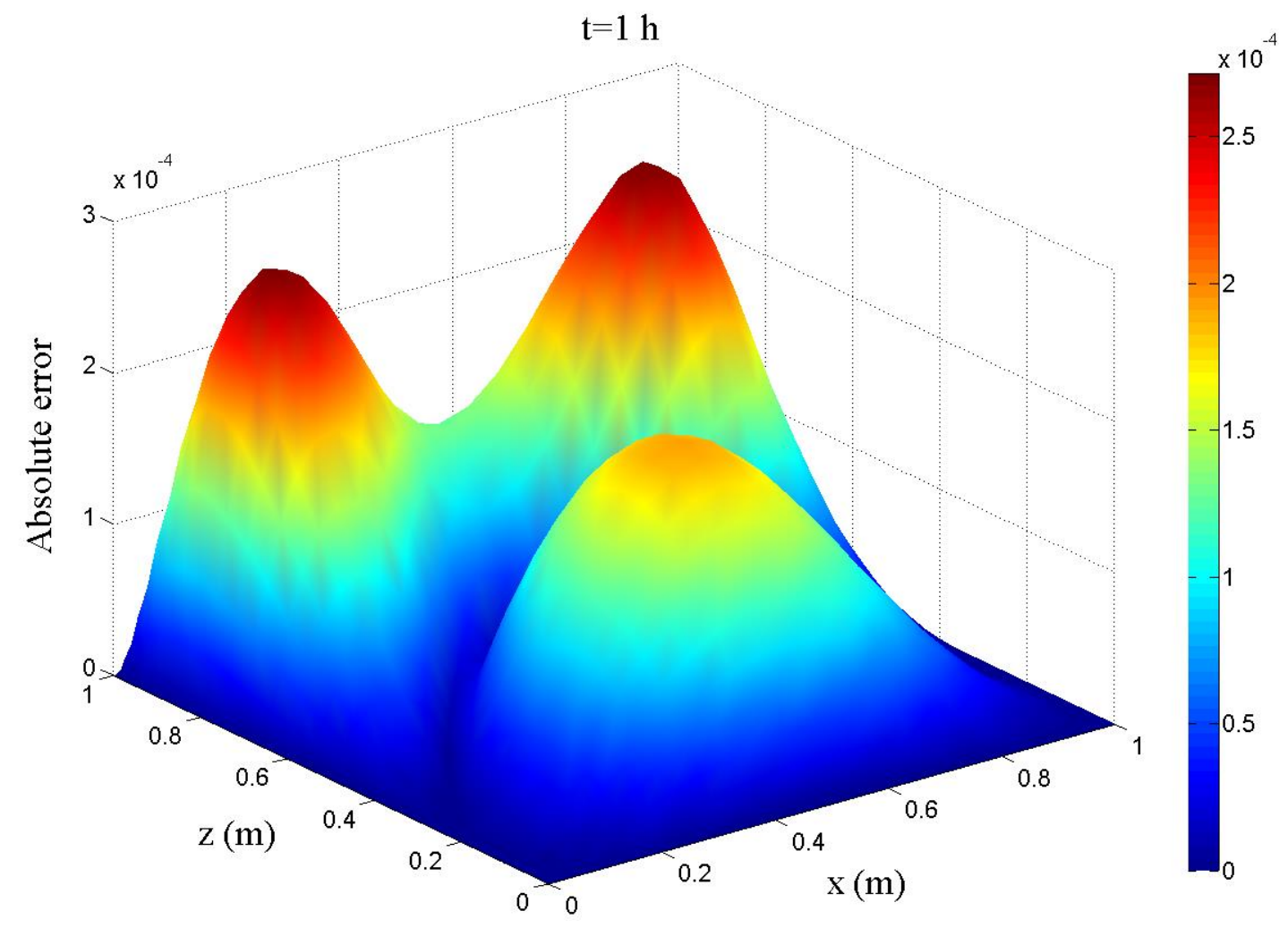

Figure 17. Absolute error of the results computed using MP(3)-ARPI relative to analytical solutions for 2D transient flow problem at $t=1 \mathrm{~h}$. 


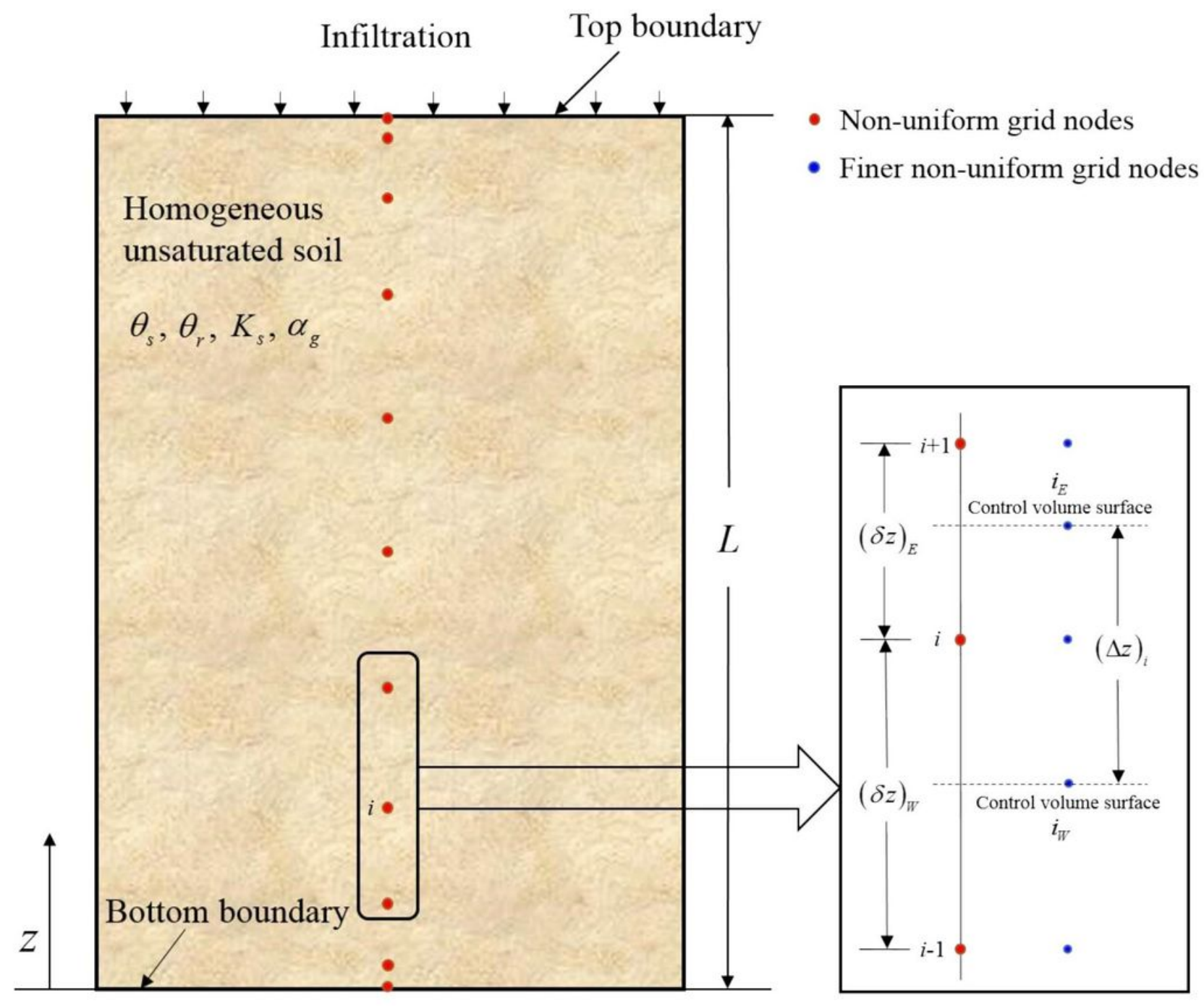

Figure 1

Schematic drawing of control volume method based on non-uniform grid nodes and 1D infiltration model for homogeneous soil. 


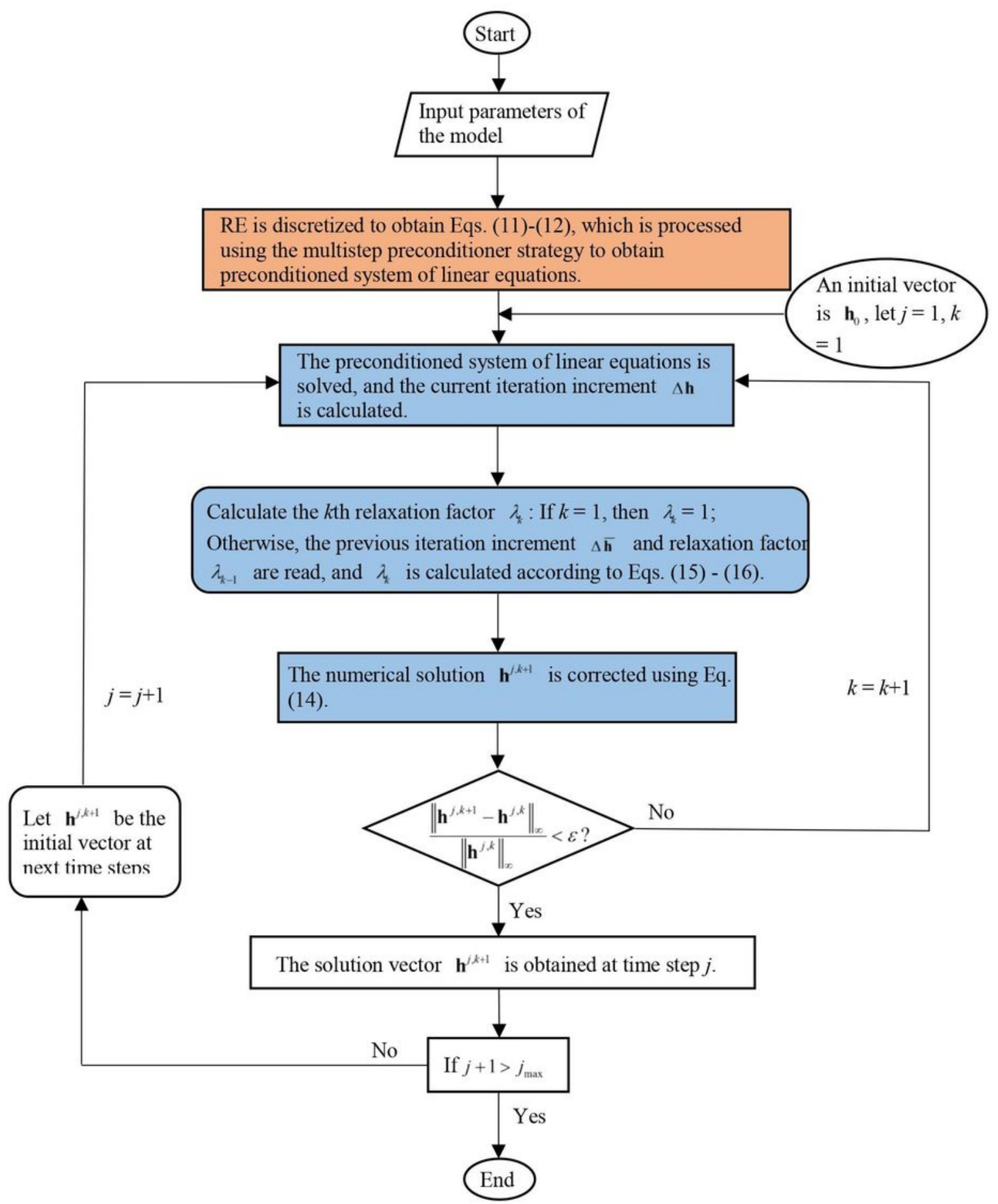

Figure 2

Brief flowchart of MP(m)-ARPI. 


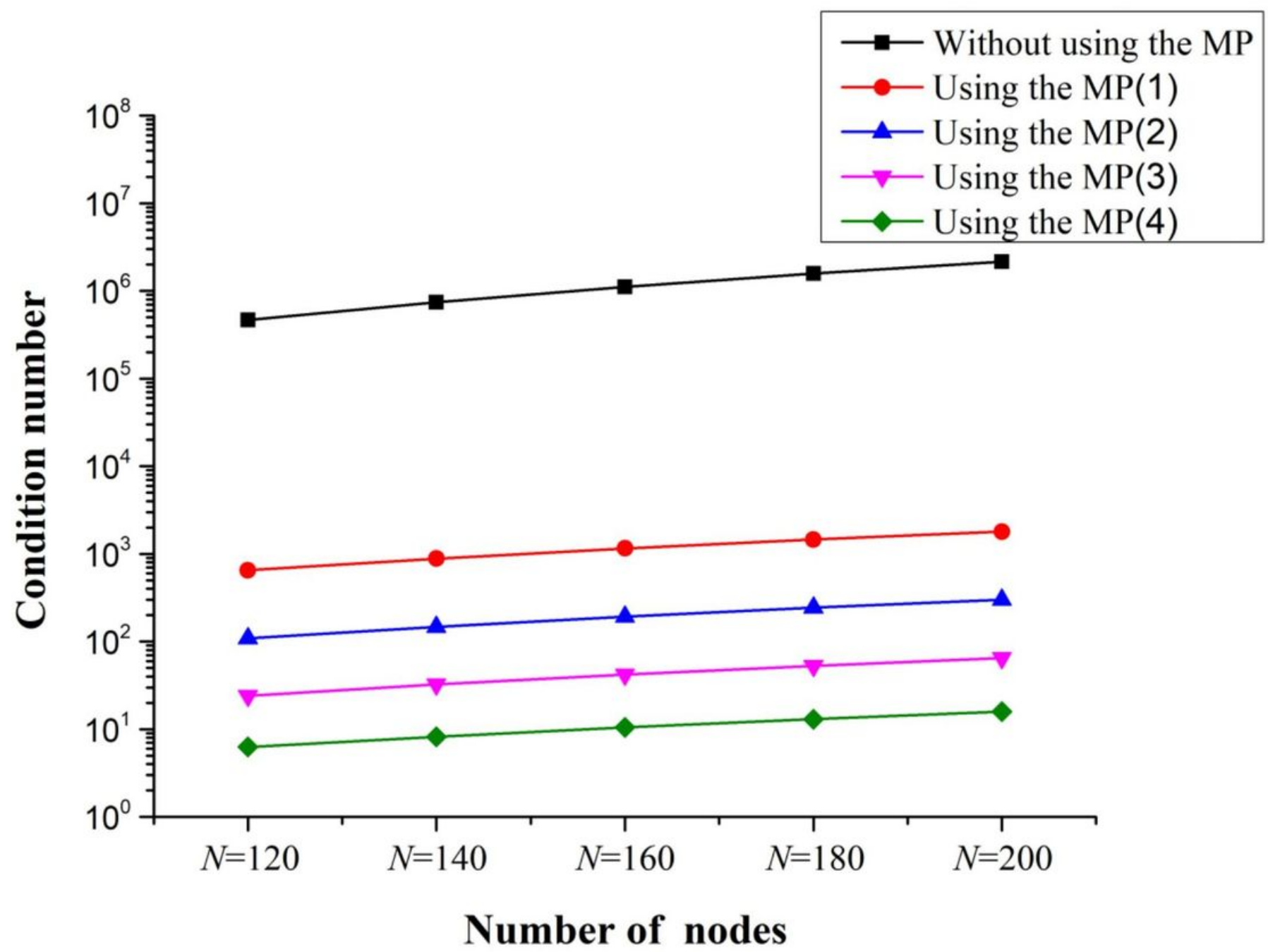

Figure 3

Condition number with and without using MP algorithm for different numbers of nodes. 


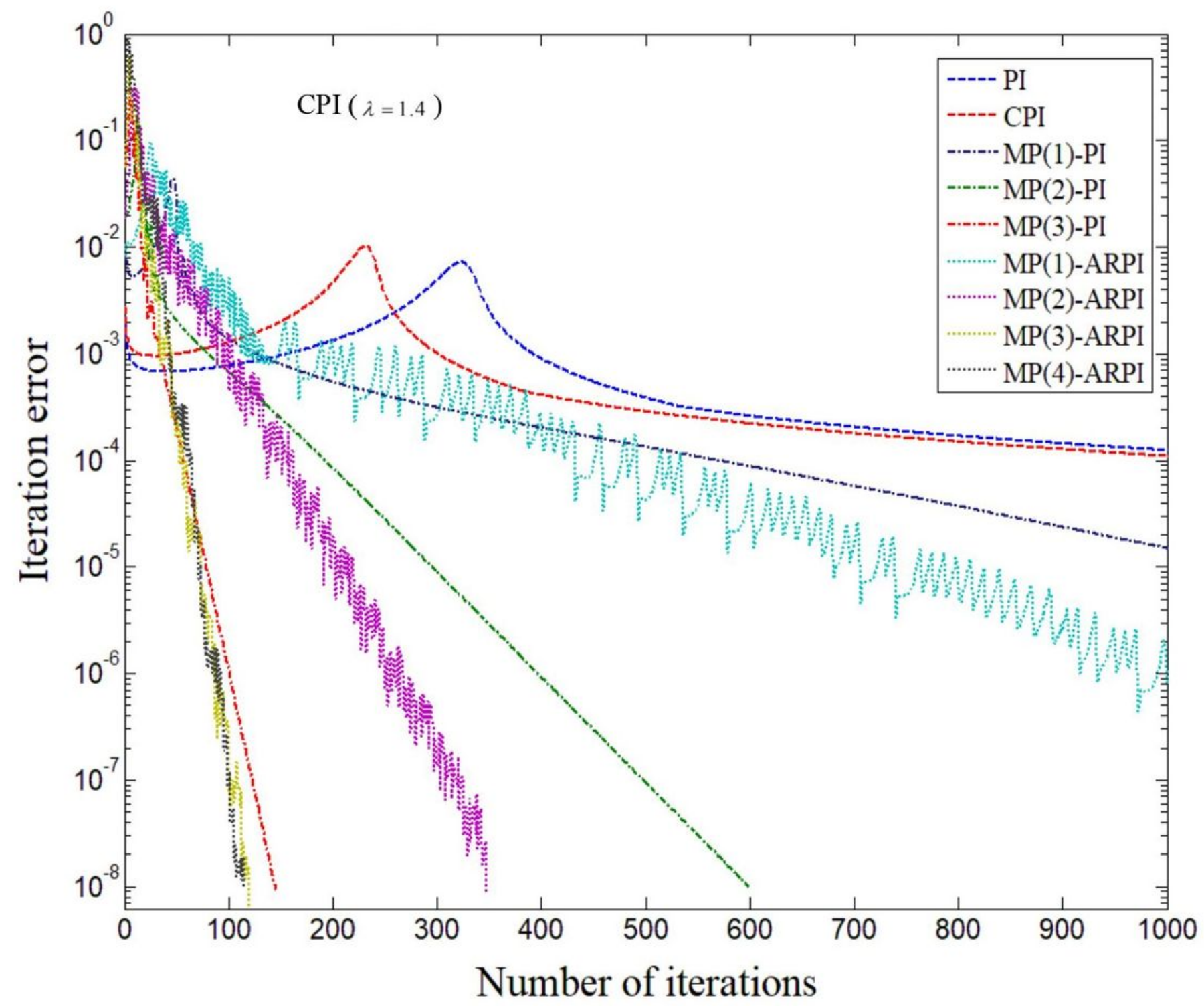

Figure 4

Comparison of convergence rates for the proposed schemes. 


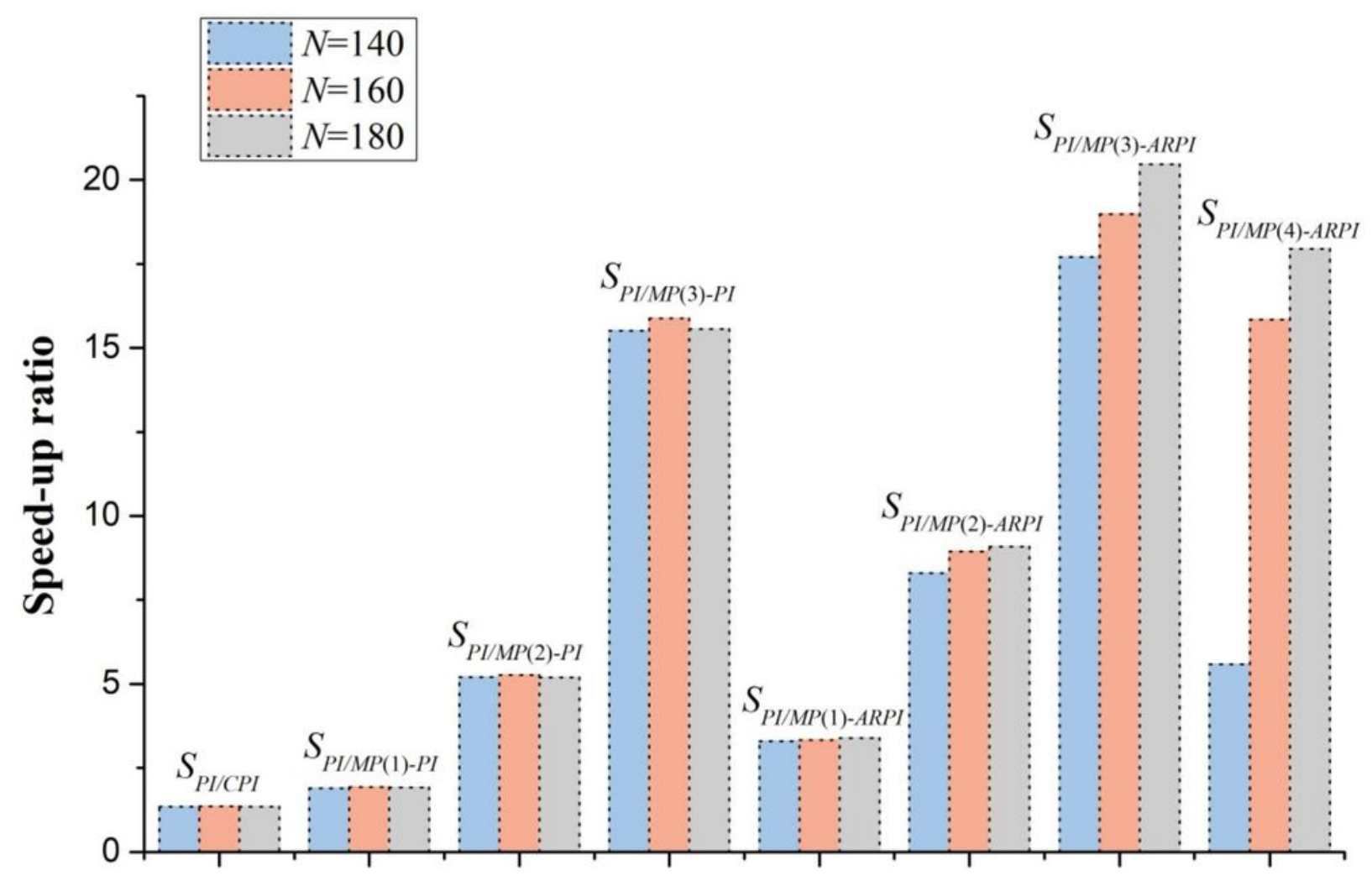

Figure 5

Comparison of speed-up ratio for the proposed schemes relative to PI. 


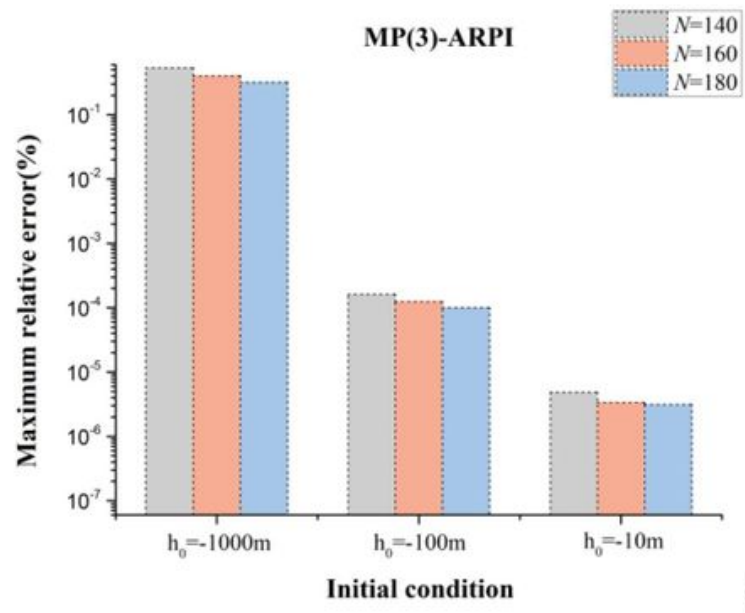

(a)

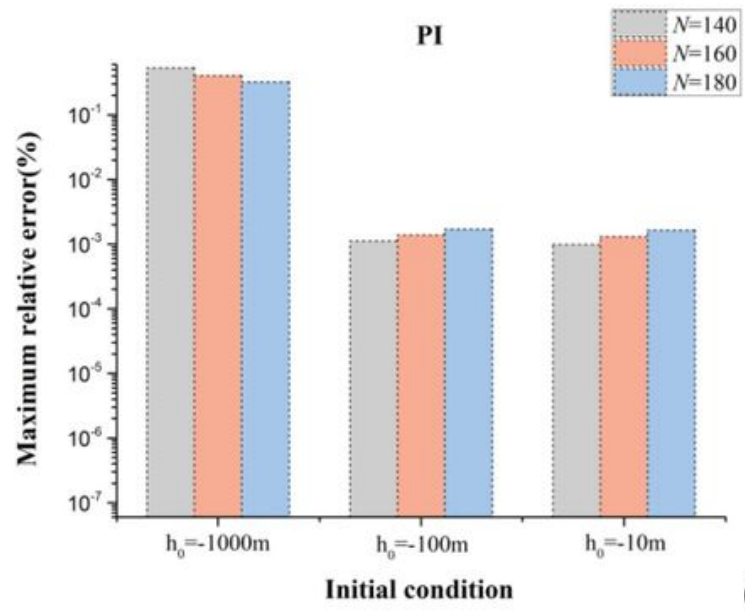

(b)

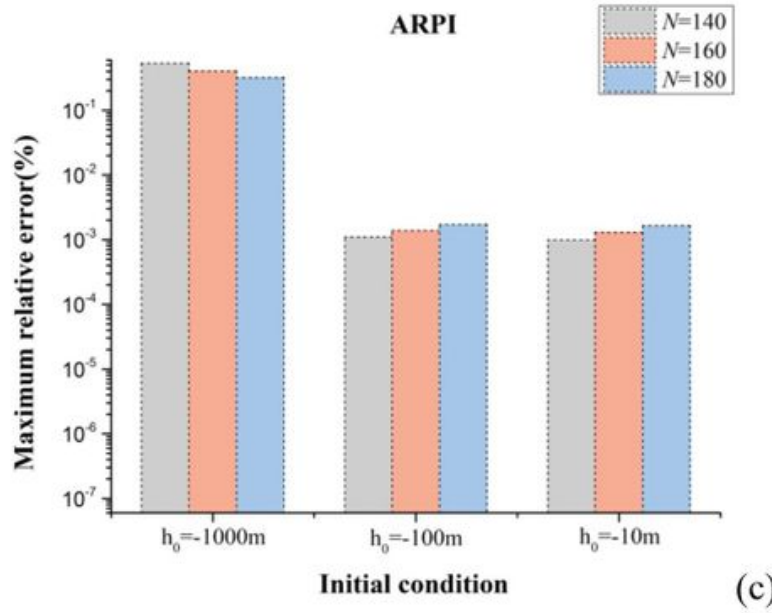

Figure 6

Comparison of the maximum relative error (\%) for different numbers of nodes and initial conditions using (a) MP(3)-ARPI, (b) PI and (c) ARPI. 


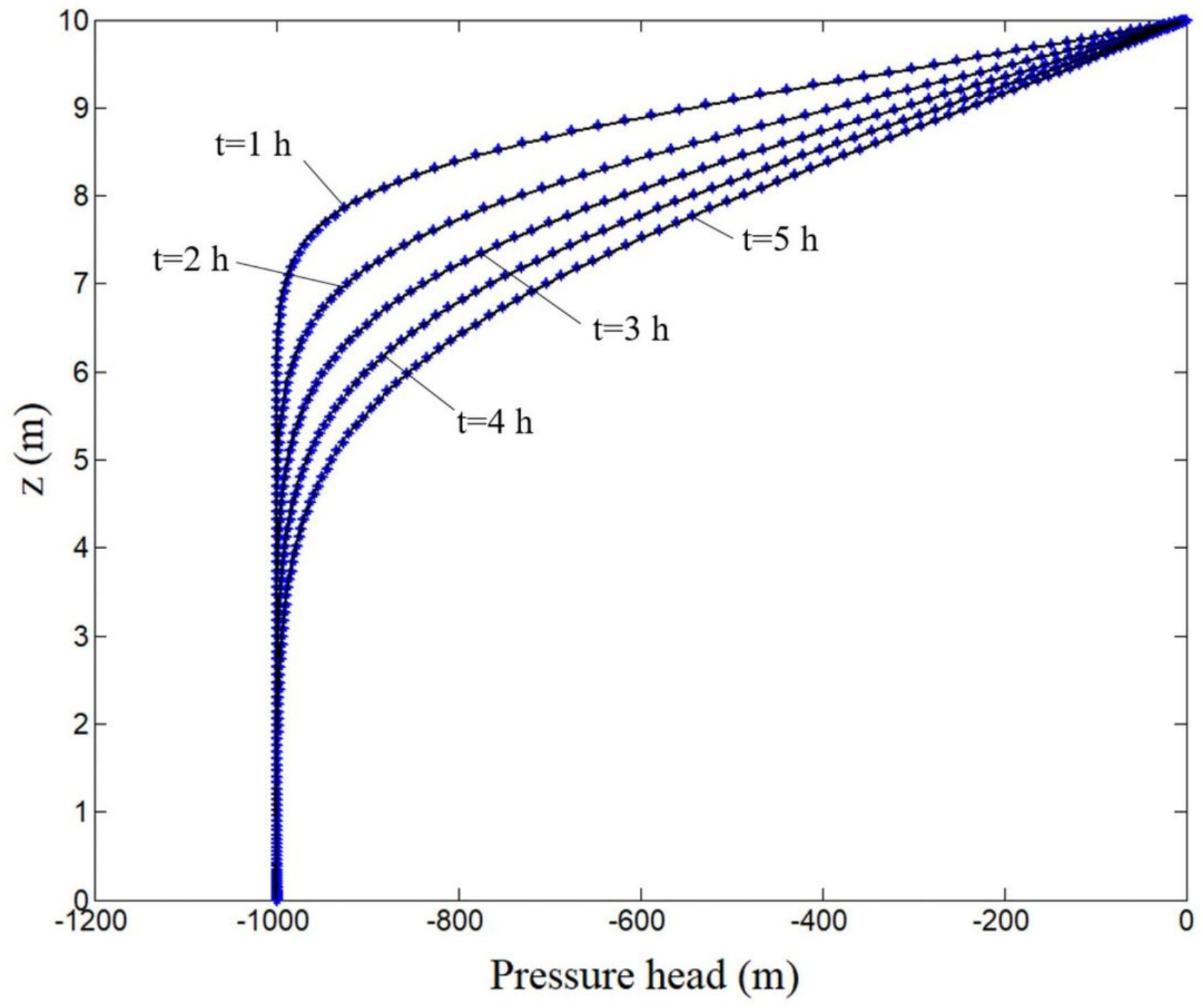

— Analytical solution (Tracy, 2006)

*** * Numerical solution by MP(3)-ARPI

Figure 7

Comparison of the numerical solutions obtained using MP(3)-ARPI with analytical solutions for Example 1. 


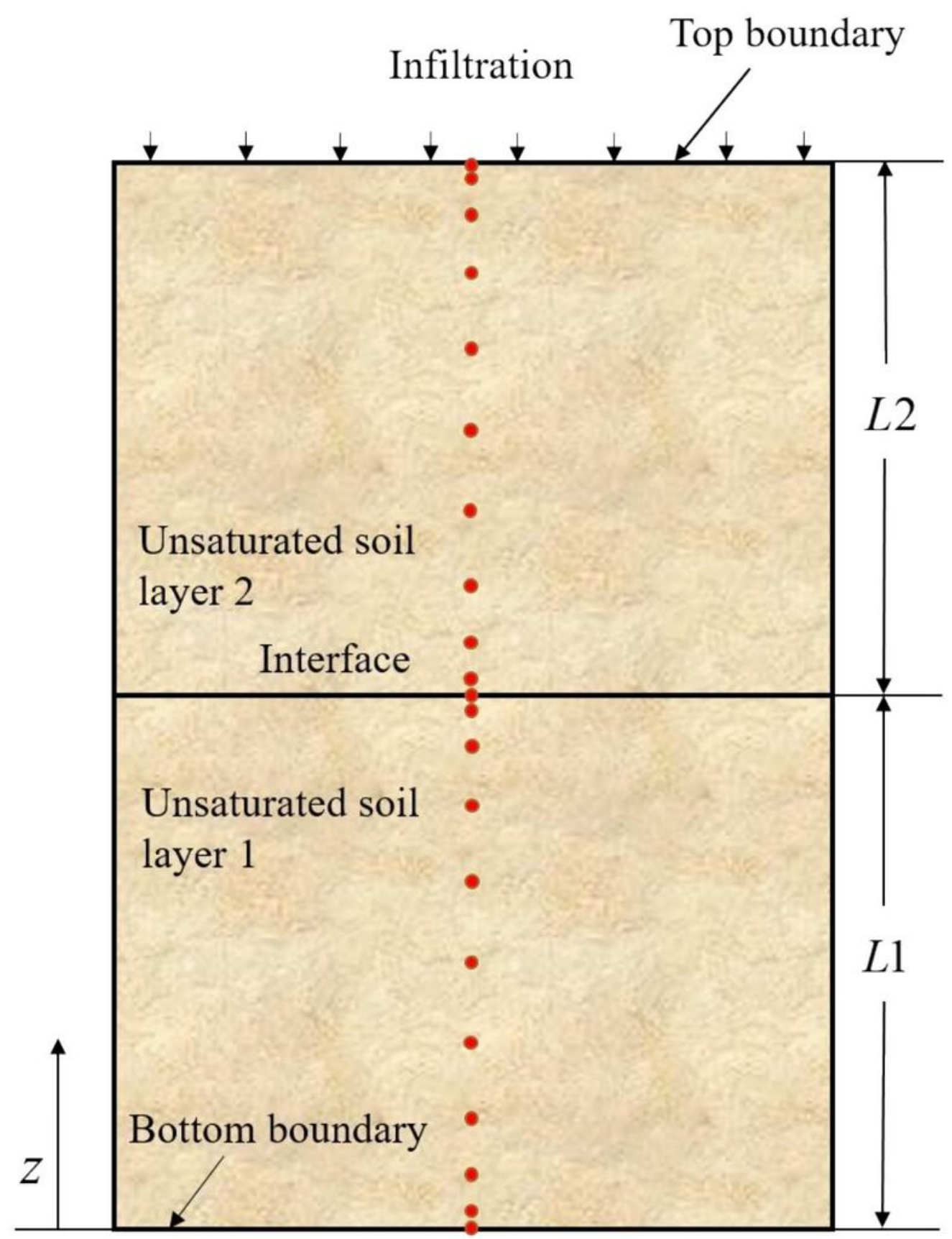

- Non-uniform grid nodes

Figure 8

1D infiltration model of two-layer unsaturated soils. 


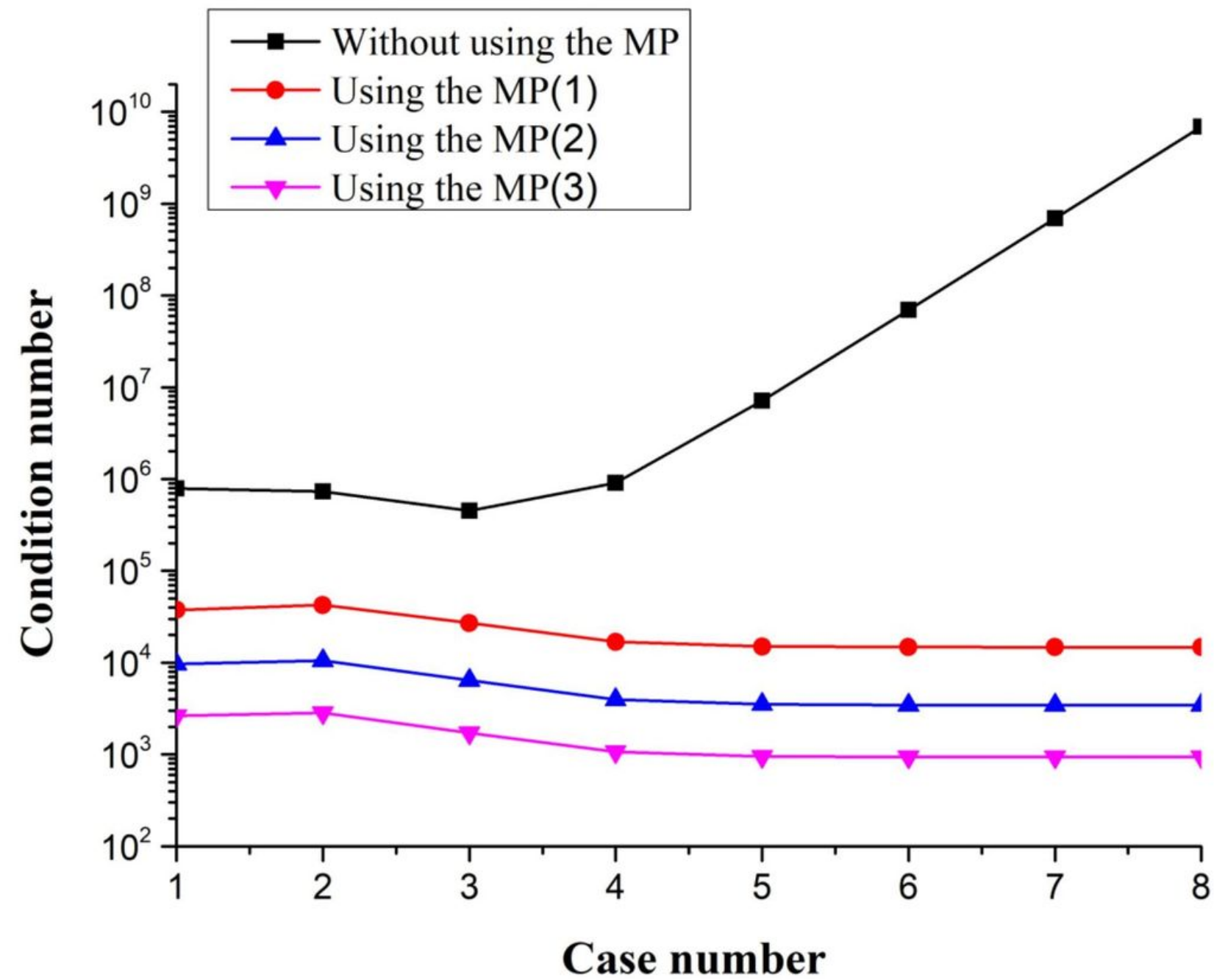

Figure 9

Condition number for Cases 1-8. 


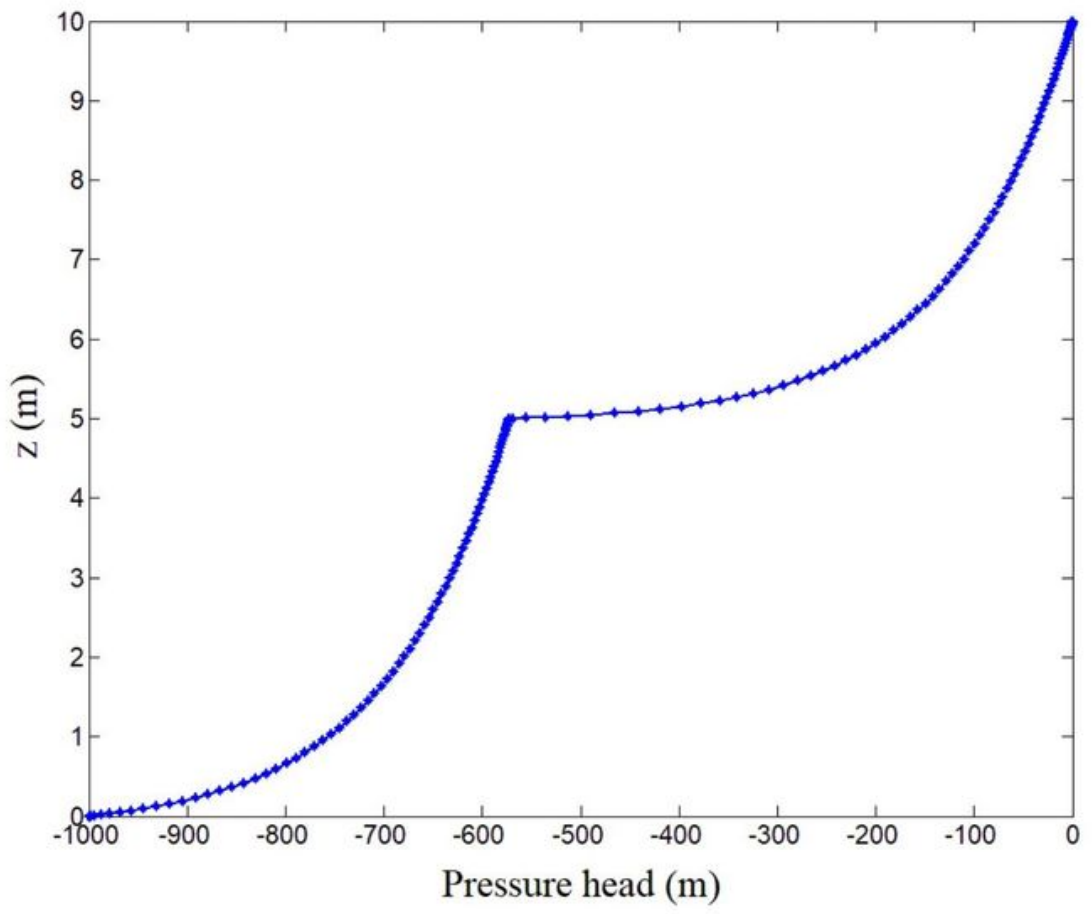

(a)

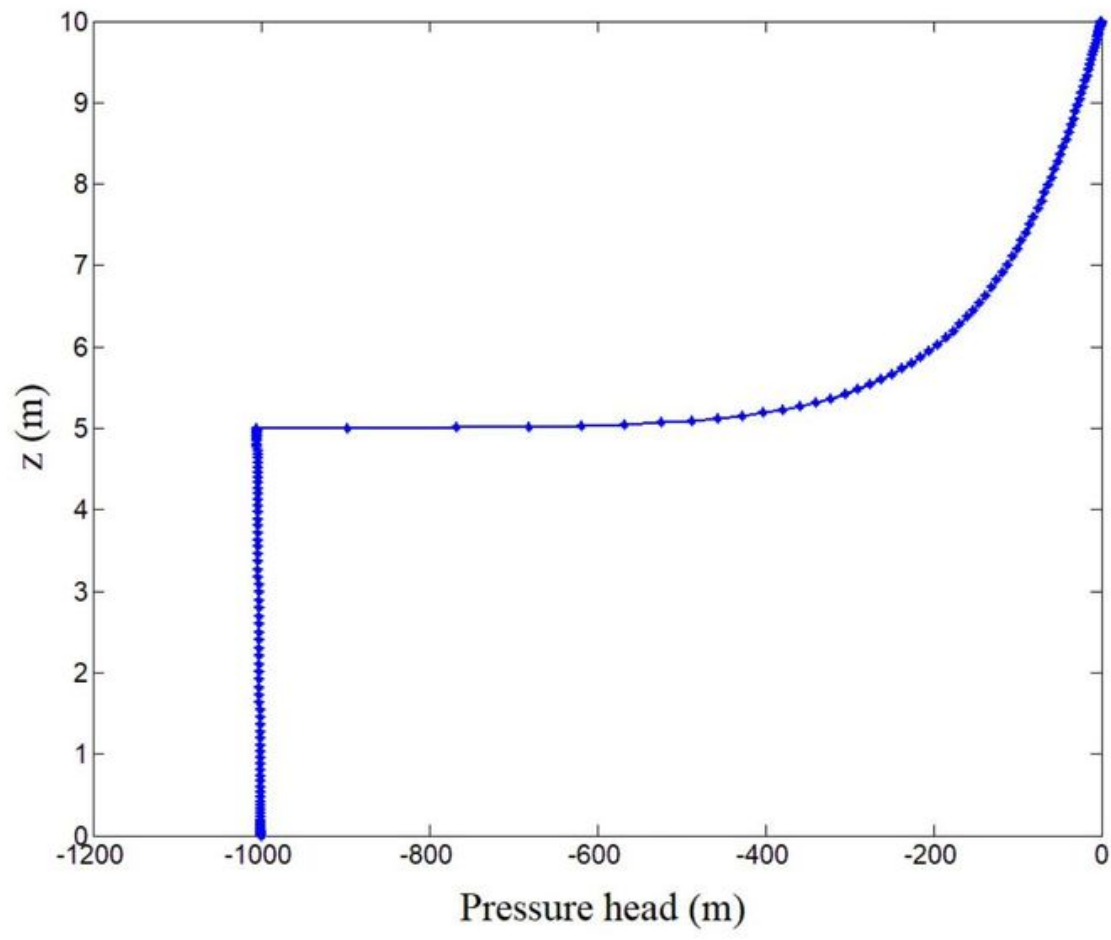

(b)

Figure 10

Numerical solutions obtained using MP(3)-ARPI: (a) Case 2; (b) Case 6. 


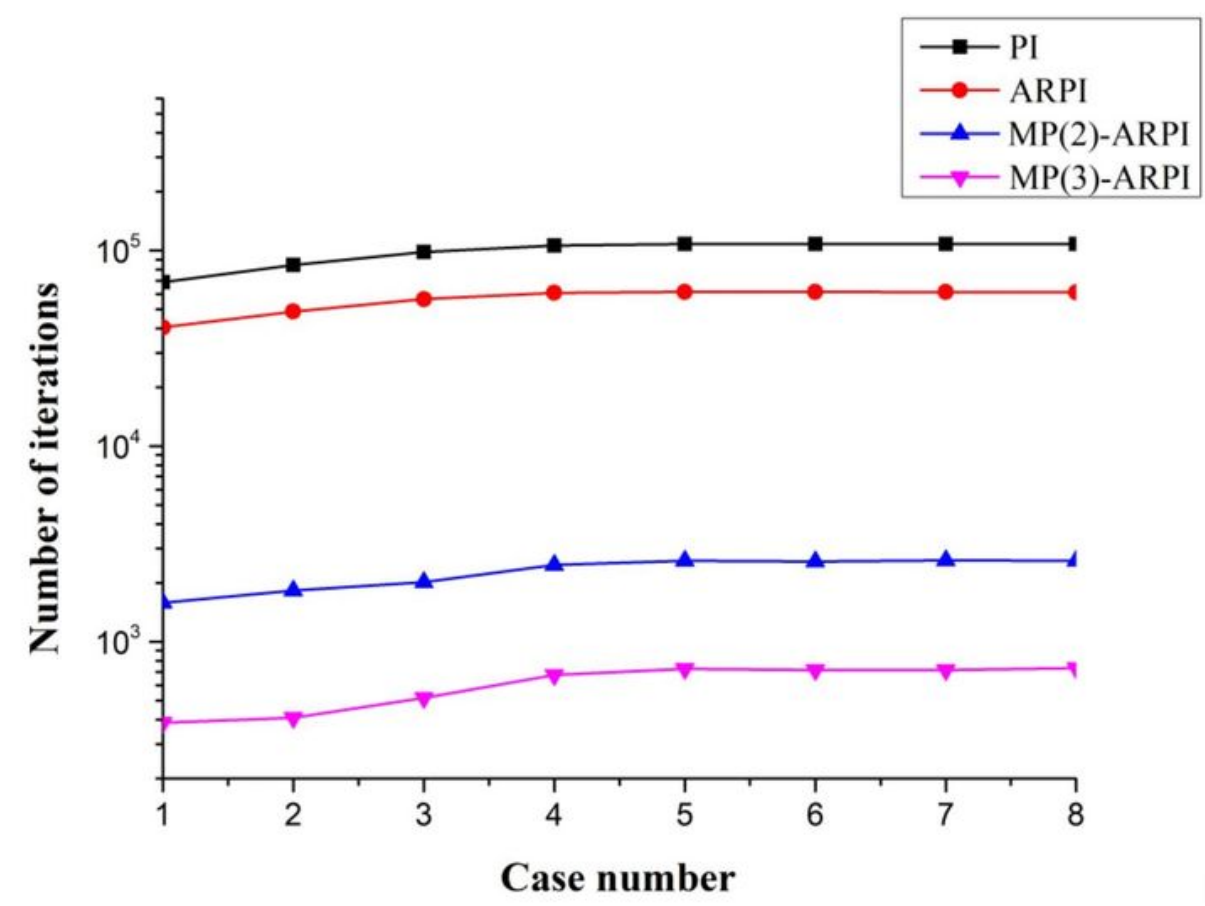

(a)

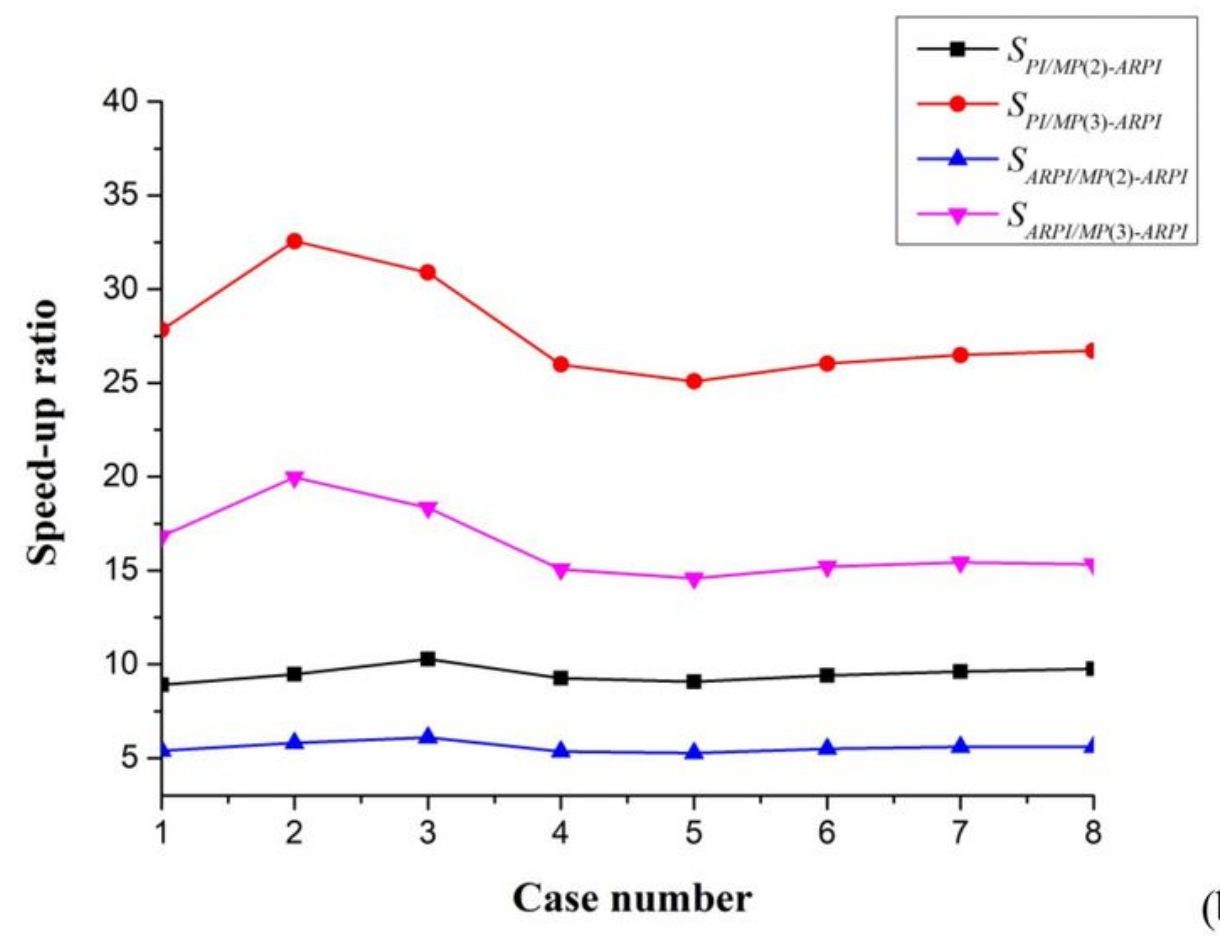

Figure 11

Numerical results for Cases 1-8: (a) Number of iterations; (b) Speed-up ratio. 


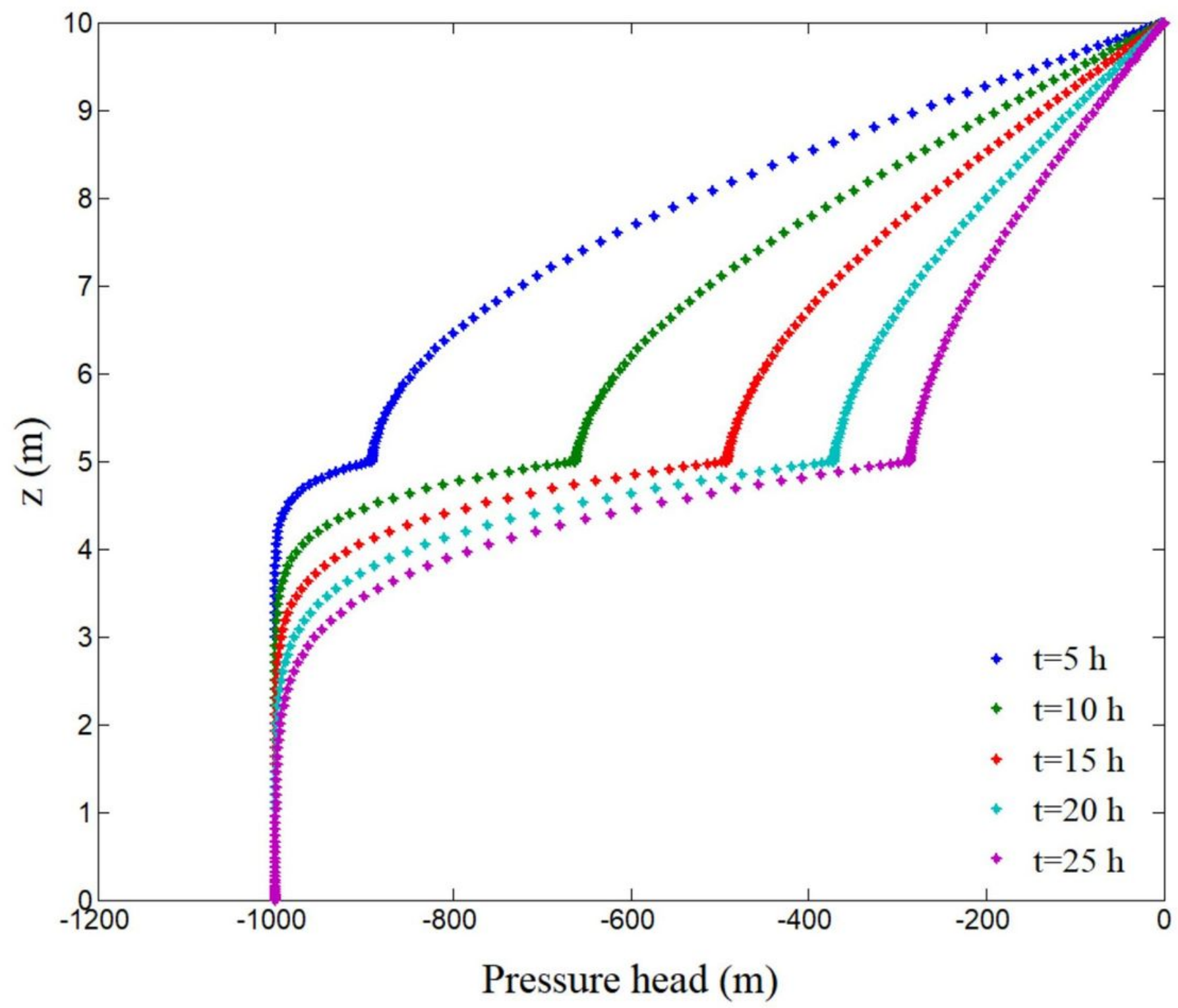

Figure 12

Numerical solution for 1D transient unsaturated flow in two-layered soils. 


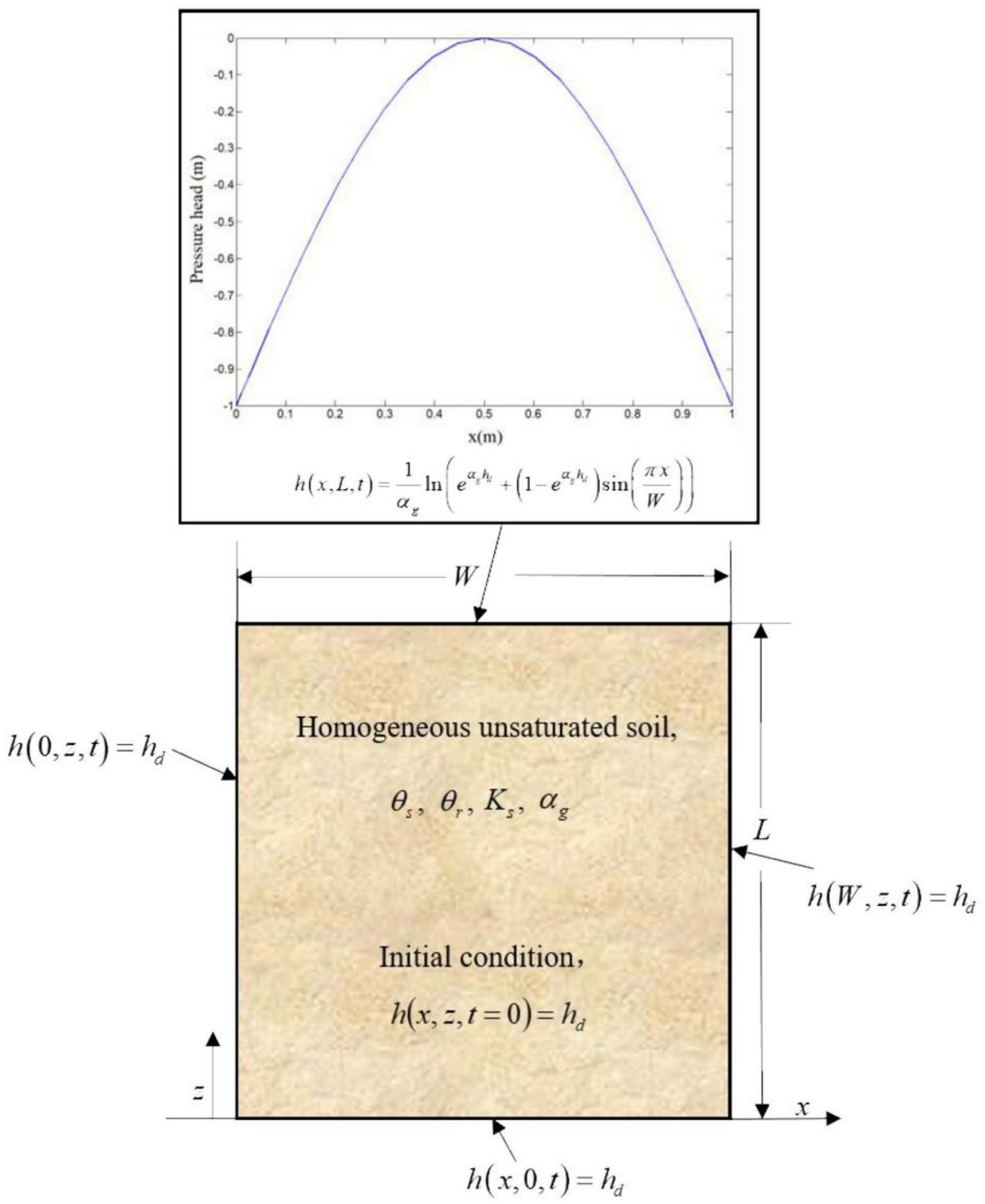

Figure 13

Mathematical model of a 2D transient flow. 


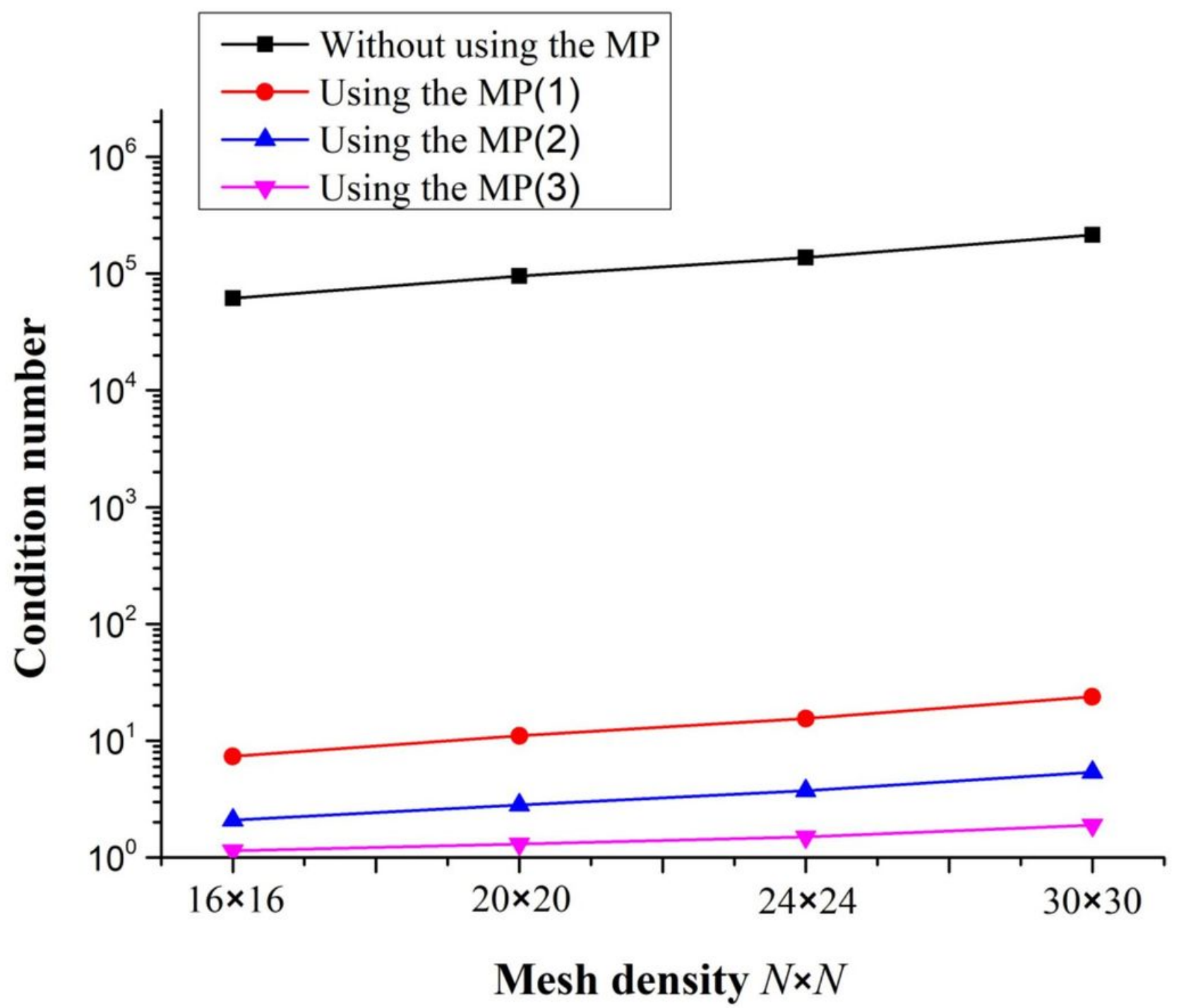

Figure 14

Condition number for different mesh densities. 

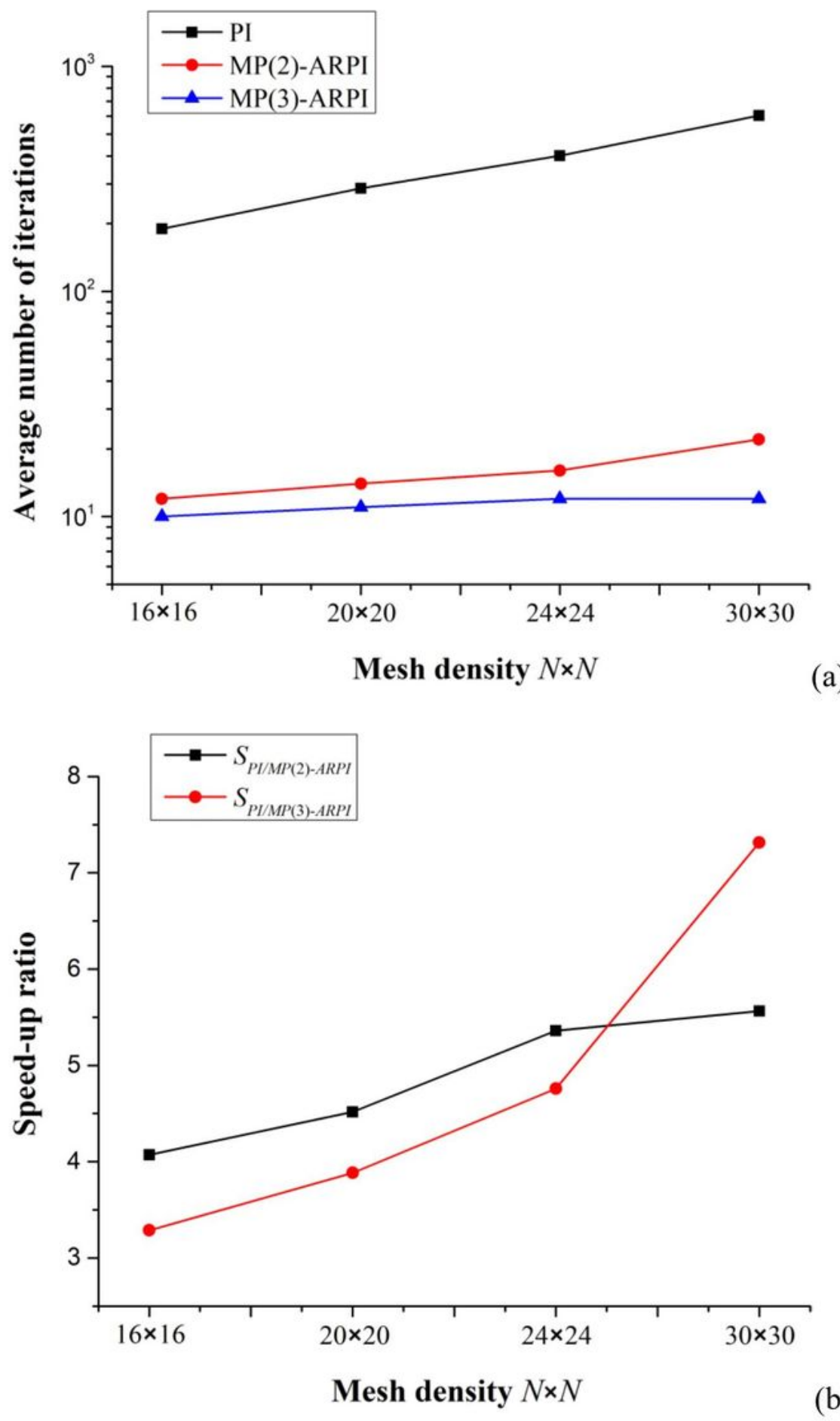

Figure 15

Numerical results for the 2D transient flow problem: (a) Average number of iterations; (b) Speed-up ratio. 


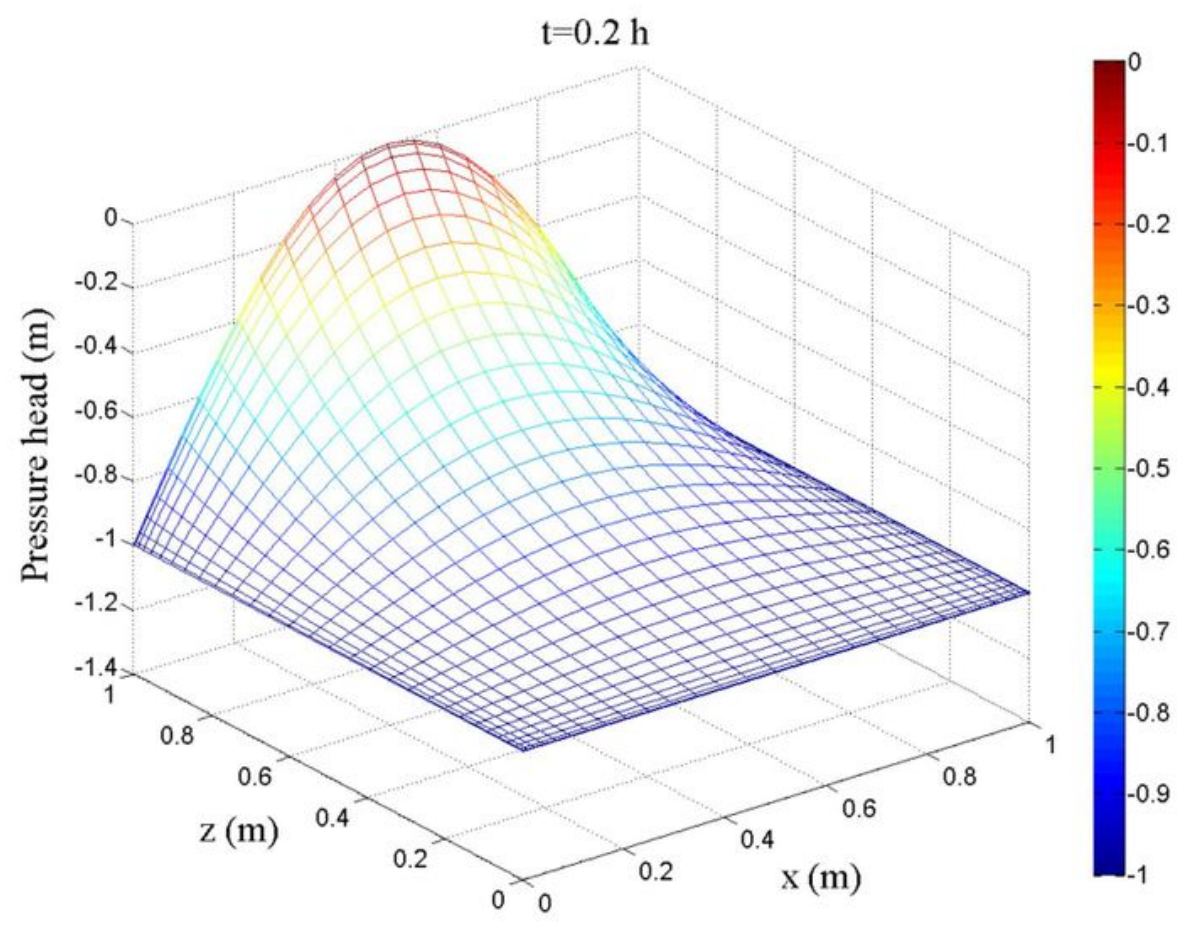

(a)

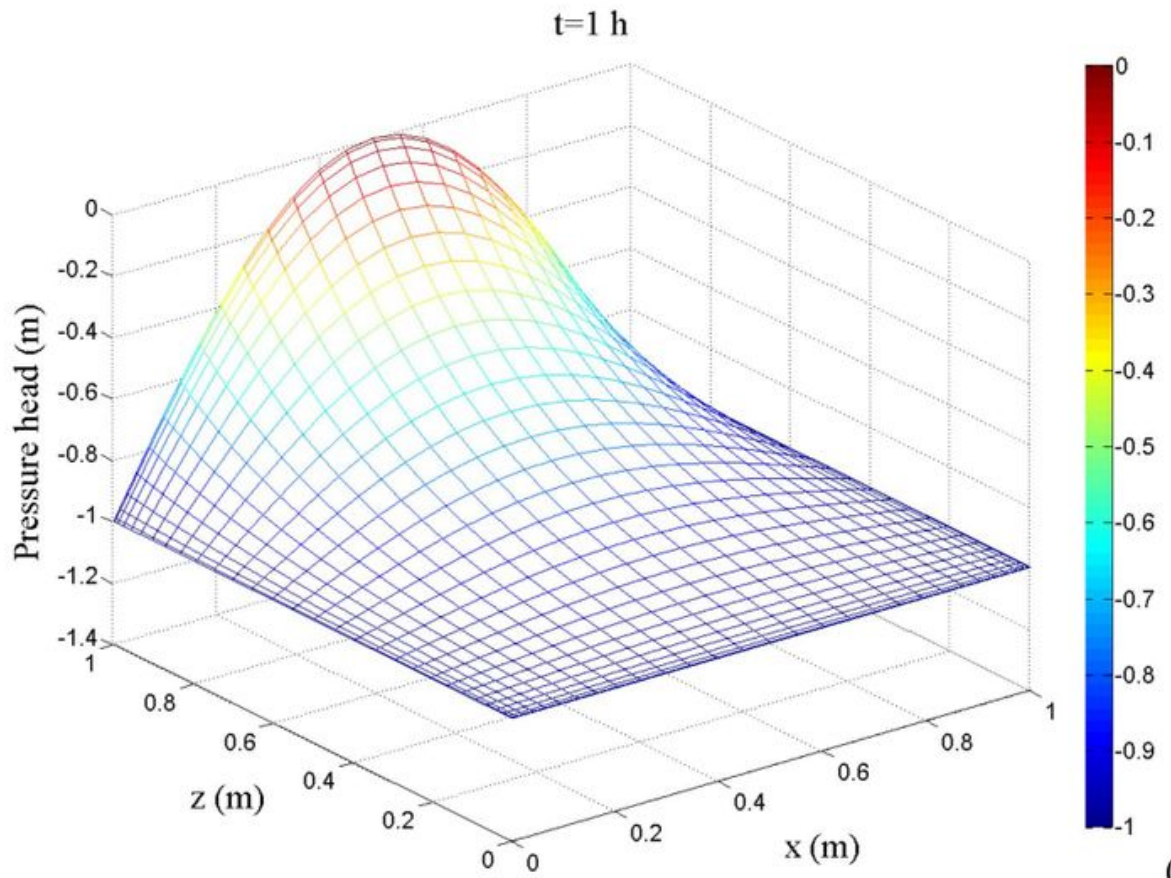

(b)

Figure 16

Numerical solutions obtained using MP(3)-ARPI for 2D transient flow problem at (a) $0.2 \mathrm{~h}$, (b) $1 \mathrm{~h}$. 


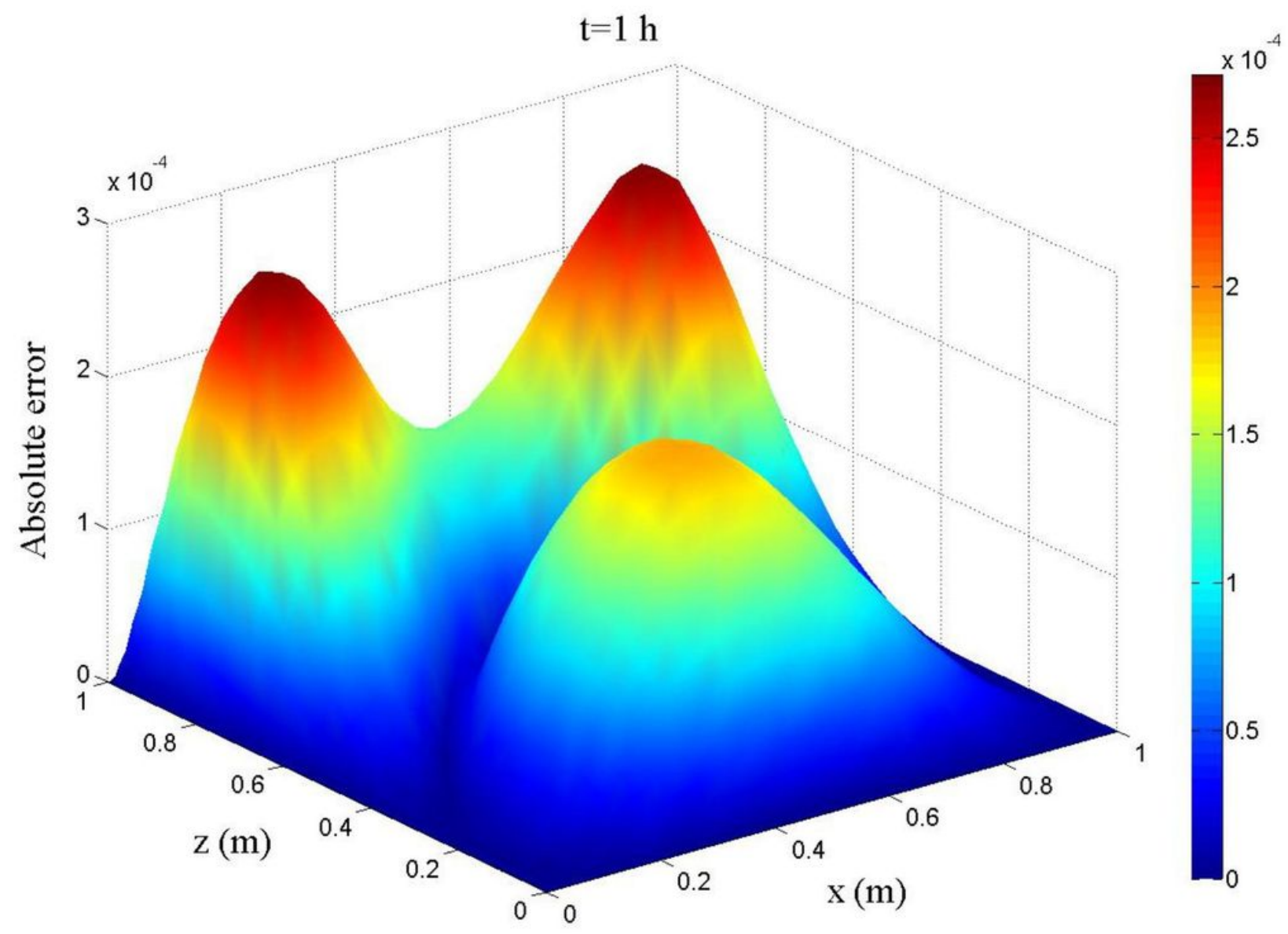

Figure 17

Absolute error of the results computed using MP(3)-ARPI relative to analytical solutions for 2D transient flow problem at $\mathrm{t}=1 \mathrm{~h}$. 